\title{
Onset of global instability in the flow past a circular cylinder cascade
}

\author{
V. B. L. Boppana ${ }^{1}$ and J. S. B. Gajjar ${ }^{2}$ \\ ${ }^{1}$ University of Southampton, School of Engineering Sciences, Highfield, Southampton, SO17 \\ 1BJ, UK \\ ${ }^{2}$ The University of Manchester, School of Mathematics, Alan Turing Building, Manchester, \\ M13 9PL, UK
}

(Received )

The effect of blockage on the onset of instability in the two-dimensional uniform flow past a cascade of cylinders is investigated. The same techniques as described in Gajjar \& Azzam (2004) are used to tackle the generalised eigenvalue problem arising from a global stability analysis of the linearised disturbance equations. Results have been obtained for the various mode classes, and our results show that for the odd-even modes which correspond to anti-phase oscillatory motion about the mid-plane between the cylinders and are the ones most extensively studied in the literature, the effect of blockage has a marginal influence on the critical Reynolds numbers for instability. This is in sharp contrast to results cited in many studies with a fully developed inlet flow past a cylinder placed between confining walls. We are also able to find other unstable modes and in particular for low blockage ratios, the odd-odd modes which correspond to in-phase oscillatory motion about the mid-plane between the cylinders, are the first to become unstable as compared to the odd-even modes, and with much lower frequencies.

\section{Introduction}

The stability of uniform flow past a circular cylinder has attracted much attention in the literature. The cylinder geometry is one of the simplest of the bluff-body flow one can study but nevertheless contains rich dynamics at fairly low Reynolds numbers. Experiments, see for example the reviews by Coutanceau \& Defaye (1991), Williamson (1996), suggest that the steady symmetric flow past a circular cylinder loses stability at fairly low Reynolds numbers. The loss of stability is characterised by slow oscillations of the wake accompanied by vortex shedding and at even higher Reynolds numbers threedimensional effects are important. However, there are significant discrepancies between different studies on the critical parameters for the loss of stability, ranging from critical $R e_{c}$ values in the mid 30's in some experiments, Coutanceau \& Bouard (1977), to $R e_{c}=$ 48 approximately, obtained by Nishioka \& Sato (1978). Here Re is the Reynolds number based on cylinder diameter and $R e_{c}$ is the critical Reynolds number for loss of stability.

An examination of the cited critical Reynolds numbers for the loss of stability in computational studies highlight similar differences with $R e_{c}=42$ reported in Strykowski \& Hanneman (1991) to $R e_{c}=54$ noted by Noack \& Eckelmann (1994). Of course in computational work differences in numerical methods and other factors does play a role in determining accurate values and some of the earlier studies may have been handicapped by inadequate computing resources in obtaining accurate values.

In this paper we consider the onset of instability in the two-dimensional uniform flow 


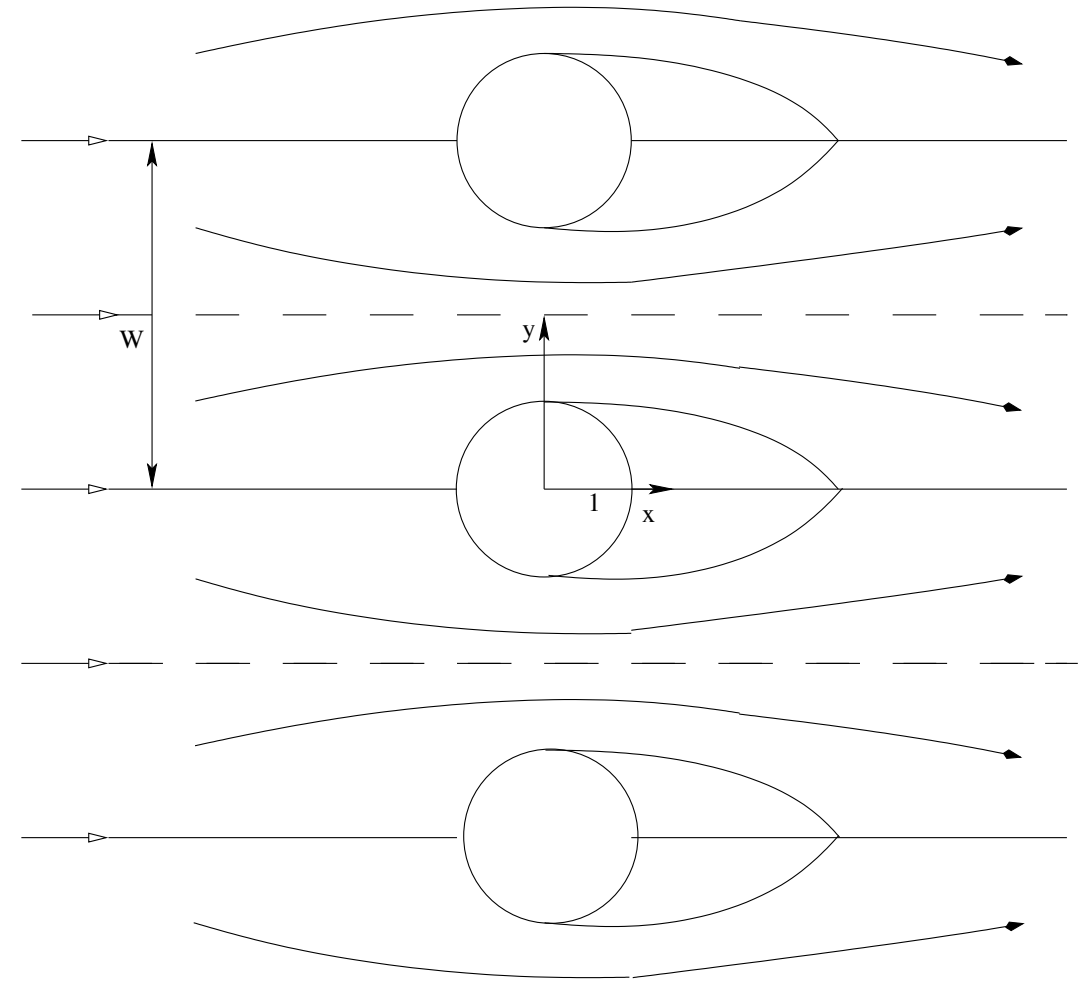

FIGURE 1. Sketch of the flow past a circular cylinder cascade.

past a cascade of cylinders. The steady case was first studied by Fornberg (1991). The flow configuration is as shown in Fig. 1. In a recent paper Gajjar \& Azzam (2004), a robust numerical method was presented for computing the steady flow and a detailed study was carried out to study the behaviour of the solution properties for large Reynolds numbers. Whereas the steady flow properties are of limited theoretical interest, especially in the limit of the Reynolds number becoming large, the onset of instability and the unsteady solution properties are of major concern. Our objective in the current work is to investigate the onset of instability for the same flow configuration using a method based on global stability analysis. In this approach the disturbances to the steady flow are assumed to be proportional to $e^{\lambda t}$ and the linearised unsteady equations are solved to determine the eigenvalues $\lambda$ as a function of the Reynolds number and blockage ratios. The blockage ratio is defined as the ratio of the cylinder diameter to distance between the cylinder centres. The onset of instability is characterised by eigenvalues crossing the real axis from negative $\Re(\lambda)$ to positive $\Re(\lambda)$. A similar approach was used by Zebib (1987) and Jackson (1987), to obtain results from a global stability analysis for the uniform flow past a circular cylinder. Zebib (1987) used a spectral method with Chebychev polynomials as basis functions to first compute the steady flow and formulated an eigenvalue problem for disturbances by decomposing the perturbations as sine and cosine expansions in the azimuthal direction. This resulted in a generalised eigenvalue problem. In Jackson (1987) the finite element method was used to solve an extended set of equations for detecting Hopf bifurcation for a class of bluff bodies. The global stability approach has also been used by Morzyński \& Thiele (1991), Morzyński et al. (1999), Chen et al. (1995), Kumar \& Mittal (2006), Mittal et al. (2008), Kumar et. al. (2009) amongst others, for discussing the instability of the flow past cylinders and related problems. Morzyński et al. (1999) was 
one of the first papers to suggest the use of subspace iteration, see Saad (1989), for solving the eigenvalue problem of the type arising in hydrodynamic stability computations using the global stability approach.

There are many other studies, see for example Strykowski \& Hanneman (1991), in which the unsteady equations are solved numerically and data from the simulations is used to estimate critical parameters for instability. In general such computations are difficult to perform accurately, but the one advantage of simulations is that a variety of initial conditions and disturbances can be explored, and also the nonlinear development can be more readily followed. Barkley \& Henderson (1996) have analysed the two-dimensional periodic wake behind a circular cylinder for Reynolds numbers between 140 and 300 and concluded that the flow becomes unstable to three-dimensional perturbations at a Reynolds number of 188.5 in line with values observed in some experiments.

In both the global stability analysis as well as the direct simulation approach, one does not need to make assumptions about the parallel or non-parallel nature of the base flow. However, both approaches lead to challenging computational problems, and their solution requires the use of efficient algorithms. The review by Theofilis (2003) contains many examples of the use of the global stability approach for investigating the stability of two- and three-dimensional flows. Recently, Marquille \& Ehrenstein (2003), Gallaire et al. (2007), have studied the instability of separated boundary layer flows over humps and have shown how a global stability analysis can be used to predict the low-frequency motion in the self-sustained oscillations generated in the wake region.

To predict the critical parameters for the onset of instability, one needs to be able to compute the steady flow accurately. But as Fornberg (1985), Fornberg (1991) has shown, for the flow past a circular cylinder this is in general a difficult task, especially if the Reynolds number is large, and proper care needs to be exercised in the choice of the method, the boundary conditions, size of domain and so on. Such considerations raise doubts about whether the size of the domain used in the work of Jackson (1987) (a maximum of 15 cylinders in the streamwise direction and 5 in the normal direction), is sufficient. In Kumar \& Mittal (2006) the finest grids used extend to 100 cylinder diameters in both directions.

Chen et al. (1995) have argued that it is difficult to compare computational results for the flow past a cylinder in an unbounded domain with experimentally obtained values, since in experiments the cylinder is usually placed between confining walls. Hence in their experimental and computational work they consider the cylinder confined between parallel plates but with a fully developed oncoming flow. This bypasses some of the difficulties in having to compute with a large domain in two directions. A similar configuration is used in the work by Sahin \& Owens (2004), and they were able to highlight the interesting complex dynamics and bifurcations especially at large blockage ratios. Camarri \& Giannetti (2007) have studied the effects of vorticity in the incoming flow and blockage effects with a square cylinder, and they suggest that the incoming flow vorticity has an important role to play in the inversion of the von Kármán street in the wake. Shair et al. (1963) had concluded from their experiments that a blockage ratio of 0.2 led to increases of over $300 \%$ in the critical Reynolds numbers. On the other hand, the developing flow between parallel plates is not the same as that of uniform flow past a cylinder and the conclusions obtained may not be appropriate for the flow geometry that is studied in the current work.

The problem studied in this paper is closely related to that discussed by Kumar \& Mittal (2006) although the numerical techniques used are different. In their work the eigenvalue problem is solved and the results are cross-checked with direct simulations of the unsteady equations. They find that the critical Reynolds number for the onset 
of instability first decreases and then increases with blockage ratio and that the critical frequencies at onset are also sensitive to the blockage ratio. This is in contrast to the trend reported in the experiments of Shair et al. (1963) and Coutanceau \& Bouard (1977). In more recent work Mittal et al. (2008) imposed centreline symmetry and studied the onset of shear layer instability in the flow past a cylinder. They find that the separated shear layer loses stability at low Reynolds numbers with very small growth rates. In Kumar et. al. (2009) the centerline symmetry condition is also used to investigate instabilities in geometries identical to the present study.

Whilst global stability analysis and direct computations are difficult to perform, especially when one is interested in accurately resolved computations with fine grids, there are many quasi-theoretical studies in which the stability properties of the wake behind a circular cylinder and other bluff bodies is investigated based on using either model wake velocity profiles, Hultgren \& Aggarwal (1987), or those from numerical simulations as in the work of Yang \& Zebib (1989) and more recently Castro (2005). In these studies the parallel flow approximation combined with the solution of the Orr-Sommerfeld or related equations, is used to estimate parameters for the onset of global instability. There have been many other attempts also to try and predict global frequencies based on local absolute instability characteristics, see for example, Pier \& Huerre (2001), Pier (2002), for two-dimensional flows and Pier (2008) for the instability in the wake of a sphere. Although the results based on such approximations may be useful in certain contexts, they are open to criticism because the wake flow behind the cylinder is clearly non-parallel. Pier (2002) has noted that the discrepancy between the predicted frequency from such an analysis and observed values for Reynolds numbers less than 100 was probably due to the non-parallelism of the near wake flow. Moreover, as Davies \& Carpenter (2003) have shown for the flow over a rotating disk, the inclusion of non-parallel effects can give rise to conclusions different from those based on a purely parallel flow analysis as put forward by Lingwood (1995). Even though there is good agreement between the experiments of Lingwood (1995) and numerical simulations of Davies \& Carpenter (2003) in the early stages of the development of the instability, the numerical simulations of Davies \& Carpenter (2003) show that this is not sustained for longer times and the rotating disk boundary layer does not produce a globally amplified mode when the flow is taken to be non-parallel.

Finally it is worth mentioning that in recent investigations of the instability of separated flows in channels, and over backward facing steps, see inter alia Marquet et. al. (2008), Blackburn et. al. (2008), it is suggested that non-modal instability, i.e. large transient growth, has a more significant role to play in the appearance of unsteadiness than say modes induced by global instability. Indeed, the relevance of global stability analysis to describe the flow dynamics observed in experiments or numerical simulations has been criticised in these studies. In Abdessemed et. al. (2009) it is argued that large transient growth effects occur and are more important than global eigenmodes, especially for the two-dimensional flow past a circular cylinder. Whilst this may be true in certain contexts, and there are many counter examples, it is our view that without a detailed and accurate study, one cannot immediately draw such conclusions about the flow in the current context. Careful numerical studies as in Mittal et al. (2008) in their investigation of the onset of shear layer instability in the flow past a cylinder, show good agreement between simulations and predicted global frequencies. In Abdessemed et. al. (2009) the computational domain extends to a lateral extent of 12.5 cylinder diameters, and streamwise extent of approximately 100 cylinder diameters. and Dirchlet conditions are imposed on the boundaries for the perturbations. Taking such a reduced domain, especially in the 
$\mathrm{C}$ $y=W / 2$

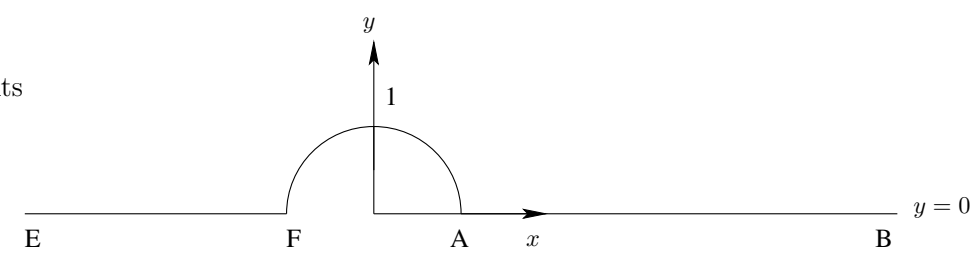

FIGURE 2. Sketch of the physical domain.

lateral direction, can significantly influence the computations and account for spurious results.

In section 2 we discuss the problem formation and governing equations, followed by brief description of the numerical techniques used in section 3 . The results of the computations for various blockage ratios are discussed in section 4 followed by our conclusions in section 5 .

\section{Problem formulation}

A circular cylinder cascade considered for the study consists of a number of circular cylinders with a unit non-dimensional radius. Their centers lie on the $y$-axis and are separated by a non-dimensional distance $W$. The blockage ratio is defined by the nondimensional parameter $\beta=2 / W$. The cascade as shown in Fig. 1 is placed in a uniform stream moving with velocity $U$ in the $x$-direction. Due to the symmetry in the $y$-direction, only the flow corresponding to $0 \leq y \leq W / 2$ is considered. The domain with the half cylinder is shown in Fig. 2.

The governing equations are the 2-D, unsteady Navier-Stokes equations for an incompressible fluid flow which when written in terms of the stream function $(\psi)$ and vorticity $(\omega)$ are:

$$
\left.\begin{array}{rl}
\nabla^{2} \psi & =-\omega, \\
\omega_{t}+\psi_{y} \omega_{x}-\psi_{x} \omega_{y} & =\frac{2}{R e} \nabla^{2} \omega .
\end{array}\right\}
$$

In Eq. (2.1), Re is the Reynolds number and is given by $R e=U d / \nu$ where $d$ is the diameter of the cylinder and $\nu$ is the kinematic viscosity of the fluid.

A detailed description of solving the steady equations (2.1) for varying $W$ and $R e$ is given in Gajjar \& Azzam (2004). The same method is adopted here to determine the flow instability. For this reason, only brief details of the method explained in Gajjar \& Azzam (2004) are mentioned in this section. Further details of the numerical methods used for both the steady and unsteady problems discussed below may also be found in Boppana (2007) or Boppana \& Gajjar (2010) where similar techniques are used to investigate the instability of flow in a lid-driven cavity.

For the ease of computations, the physical domain is as shown in Fig. 2 with the $X(=x+i y)$ domain transformed to a rectangular strip denoted by $Z(=\xi+i \eta)$. The transformed domain as indicated in Fig. 3 is obtained by means of the conformal mapping described by Fornberg (1991) and Gajjar \& Azzam (2004).

The governing equations (2.1) and boundary conditions in the transformed domain are given by: 


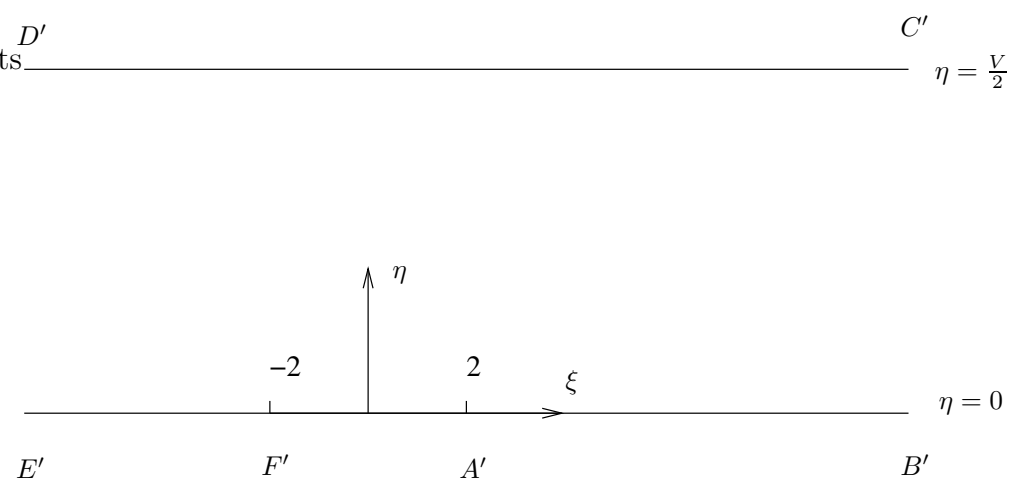

FIGURE 3. Sketch of the transformed domain.

$$
\left.\begin{array}{r}
\left\{\frac{\partial^{2} \psi}{\partial \xi^{2}}+\frac{\partial^{2} \psi}{\partial \eta^{2}}\right\} J+\omega=0 \\
\omega_{t}+\left\{\frac{2}{R e}\left(\frac{\partial^{2} \omega}{\partial \xi^{2}}+\frac{\partial^{2} \omega}{\partial \eta^{2}}\right)+\left(\frac{\partial \psi}{\partial \xi} \frac{\partial \omega}{\partial \eta}-\frac{\partial \psi}{\partial \eta} \frac{\partial \omega}{\partial \xi}\right)\right\} J=0 .
\end{array}\right\}
$$

$\psi=0, \quad \omega=0 \quad$ on $A^{\prime} B^{\prime}$ i.e, $\quad 2<\xi<\infty \quad \& \quad \eta=0$,

$\psi=0, \quad \omega=0 \quad$ on $E^{\prime} F^{\prime}$ i.e, $\quad-\infty<\xi<-2 \quad \& \quad \eta=0$,

$\psi=\frac{W}{2}, \quad \omega=0 \quad$ on $C^{\prime} D^{\prime}$ i.e, $\quad-\infty<\xi<\infty \quad \& \quad \eta=\frac{V}{2}$,

$\psi=0, \quad \psi_{\eta}=0 \quad$ on $F^{\prime} A^{\prime}$ i.e, $\quad-2 \leq \xi \leq 2 \quad \& \quad \eta=0$,

$\psi \rightarrow \eta, \quad \psi_{\xi}=0, \quad \omega=0 \quad$ on $B^{\prime} C^{\prime}$ i.e, $\quad \xi=\infty \quad \& \quad 0<\eta<\frac{V}{2}$,

$\psi \rightarrow \eta, \quad \psi_{\xi}=0, \quad \omega=0 \quad$ on $D^{\prime} E^{\prime}$ i.e, $\quad \xi=-\infty \quad \& \quad 0<\eta<\frac{V}{2}$.

where $J=|d Z / d X|^{2}$ is the Jacobian of the transformation.

\section{Hydrodynamic stability analysis}

To analyze the stability, we used the method of normal modes, according to which the total flow $(\psi(\xi, \eta, t)$ and $\omega(\xi, \eta, t))$ is expressed as

$$
\left.\begin{array}{rl}
\psi(\xi, \eta, t) & =\bar{\psi}(\xi, \eta)+\delta \widetilde{\psi}(\xi, \eta) \exp (\lambda t), \\
\omega(\xi, \eta, t) & =\bar{\omega}(\xi, \eta)+\delta \widetilde{\omega}(\xi, \eta) \exp (\lambda t) .
\end{array}\right\}
$$

and $\delta$ is a small disturbance size. Substituting (3.1) into the governing equations (2.2) gives rise to the following steady and linearized stability system of equations:

Steady equations and boundary conditions:

$$
\left.\begin{array}{r}
\left\{\frac{\partial^{2} \bar{\psi}}{\partial \xi^{2}}+\frac{\partial^{2} \bar{\psi}}{\partial \eta^{2}}\right\} J+\bar{\omega}=0 \\
\text { and } \quad \frac{\partial^{2} \bar{\omega}}{\partial \xi^{2}}+\frac{\partial^{2} \bar{\omega}}{\partial \eta^{2}}+\frac{1}{2} R e\left\{\frac{\partial \bar{\psi}}{\partial \xi} \frac{\partial \bar{\omega}}{\partial \eta}-\frac{\partial \bar{\psi}}{\partial \eta} \frac{\partial \bar{\omega}}{\partial \xi}\right\}=0 .
\end{array}\right\}
$$


Linearized stability equations and boundary conditions:

$$
\left.\begin{array}{r}
\left\{\frac{\partial^{2} \widetilde{\psi}}{\partial \xi^{2}}+\frac{\partial^{2} \widetilde{\psi}}{\partial \eta^{2}}\right\} J+\widetilde{\omega}=0 \\
\text { and } \quad-\lambda \widetilde{\omega}+\left\{\frac{2}{R e}\left(\frac{\partial^{2} \widetilde{\omega}}{\partial \xi^{2}}+\frac{\partial^{2} \widetilde{\omega}}{\partial \eta^{2}}\right)+\left(\frac{\partial \widetilde{\psi}}{\partial \xi} \frac{\partial \widetilde{\omega}}{\partial \eta}-\frac{\partial \widetilde{\psi}}{\partial \eta} \frac{\partial \widetilde{\omega}}{\partial \xi}\right)\right\} J=0 .
\end{array}\right\}
$$

The constant $V$ is determined via the conformal mapping, Fornberg (1991). Only the half domain of the flow past a circular cylinder is considered due to the flow being symmetric with respect to $\xi$ axis (i.e. $u(\xi, \eta)=u(\xi,-\eta)$. This implies $\bar{\psi}(\xi, \eta)=-\bar{\psi}(\xi,-\eta)$ and $\bar{\omega}(\xi, \eta)=-\bar{\omega}(\xi,-\eta))$ and two sets of boundary conditions arise on the lower boundary, the cylinder surface excepted. They are either

$$
\widetilde{\psi}=0 \quad \text { and } \quad \widetilde{\omega}=0, \quad|\xi|>2, \quad \eta=0,
$$

which we label as even modes (the streamwise disturbance velocity component is even about $(\eta=0))$ or

$$
\widetilde{\psi}_{\eta}=0 \quad \text { and } \quad \widetilde{\omega}_{\eta}=0, \quad|\xi|>2, \quad \eta=0
$$

which then correspond to odd modes. A similar classification can be made with respect to the boundary condition on $\eta=V / 2$ with for instance even modes given by

$$
\widetilde{\psi}=0 \quad \text { and } \quad \widetilde{\omega}=0, \quad \eta=V / 2,
$$

This gives rise to 4 mode classes which are studied below. These are even-even (EE) modes, even-odd (EO) modes, odd-even (OE) modes, and odd-odd (OO) modes, and in all cases the first letter corresponds to the boundary conditions on $\eta=0$ and the second letter to the boundary condition on $\eta=V / 2$. The OE modes are the closest to the ones studied in the flow past an isolated cylinder.

\subsection{Numerical method}

A further spline transformation in which $\xi=g(\tau)$ is used in the $\xi$ direction to attain a uniform grid in terms of $\tau$, for more details see Fornberg (1991), Gajjar \& Azzam (2004). Fourth-order central differences in the $\tau$-direction and Chebyshev collocation in the $\eta$-direction are used for discretizing the equations. In the $\eta$ direction, the grid points are located at the Chebyshev collocation nodes. The domain was truncated in the $\tau$ direction such that the lower bound of it is denoted by $\tau_{\min }$ and the upper bound by $\tau_{\max }$. Therefore, the uniform grid in $\tau$ is given by

$$
\tau_{j}=\tau_{\min }+(j-1) h, \quad \text { where } \quad j=1, \ldots M \quad \text { and } \quad h=\frac{\tau_{\max }-\tau_{\min }}{M-1} .
$$

In the $\eta$-direction, the domain is mapped to Chebyshev space i.e, $\eta \in\left[0, \frac{V}{2}\right] \rightarrow z \in[-1,1]$ where

$$
z_{k}=\cos \left(\frac{k \pi}{n}\right), \quad k=0,1 \ldots n \quad \text { and } \quad \eta_{k}=\frac{V}{4}\left(z_{k}+1\right) .
$$

The discretization of the basic flow equations gives rise to a set of discrete equations which are nonlinear. These are linearized using a Newton-Raphson technique. For this purpose, let $\bar{\psi}_{i, j}=\Psi_{i, j}+H_{i, j}$ and $\bar{\omega}_{i, j}=\Omega_{i, j}+G_{i, j}$, where $\Psi_{i, j}, \Omega_{i, j}$ are some initial guesses and $H_{i, j}, G_{i, j}$ are corresponding correction factors such that $\left|H_{i, j}\right|,\left|G_{i, j}\right|<<1$. 
Let the total number of grid points be denoted by $M$ in the $\tau$-direction and $N(=n+1)$ in the $\eta$-direction. Thus the linearized steady equations, after ignoring the smaller order nonlinear terms and collecting the like terms will be of the form

$$
\overline{\mathbf{A}}^{(p)} \boldsymbol{\Phi}_{p-2}+\overline{\mathbf{B}}^{(p)} \boldsymbol{\Phi}_{p-1}+\overline{\mathbf{C}}^{(p)} \boldsymbol{\Phi}_{p}+\overline{\mathbf{D}}^{(p)} \boldsymbol{\Phi}_{p+1}+\overline{\mathbf{E}}^{(p)} \boldsymbol{\Phi}_{p+2}=\mathbf{R}^{(p)}, \quad 1 \leq p \leq M
$$

where the matrices $\overline{\mathbf{A}}$ to $\overline{\mathbf{E}}$ are of size $2 N \times 2 N$ and they arise due to the enforcement of Chebyshev collocation nodes in the $y$-direction. $\boldsymbol{\Phi}_{p}$ is a vector of correction factors of stream function $\left(H_{p}\right)$ and vorticity $\left(G_{p}\right)$ at each node in the $\tau$-direction i.e.,

$$
\boldsymbol{\Phi}_{p}=\left(\underline{H}_{p}\right)=\left(H_{p, 0}, \ldots, H_{p, n}, G_{p, 0}, \ldots, G_{p, n}\right)^{T} .
$$

A block penta-diagonal structure arises as a result of discretizing the equations with fourth-order central difference in the $\tau$-direction. The linear system (3.4), when written in matrix form results in

$$
\overline{\mathbf{L}} \Phi=\overline{\mathbf{R}}
$$

where

$$
\mathbf{\Phi}=\left(\boldsymbol{\Phi}_{1}, \ldots, \mathbf{\Phi}_{M}\right)^{T}
$$

Equation (3.5) is solved for $\boldsymbol{\Phi}$ using a direct solver which exploits the sparsity of the block penta-diagonal structure of matrix $\overline{\mathbf{L}}$.

The obtained stationary solution is used in the linear stability equations which after discretization and collecting the like terms gives rise to

$$
\widetilde{\mathbf{A}}^{(p)} \boldsymbol{\Theta}_{p-2}+\widetilde{\mathbf{B}}^{(p)} \boldsymbol{\Theta}_{p-1}+\widetilde{\mathbf{C}}^{(p)} \boldsymbol{\Theta}_{p}+\widetilde{\mathbf{D}}^{(p)} \boldsymbol{\Theta}_{p+1}+\widetilde{\mathbf{E}}^{(p)} \boldsymbol{\Theta}_{p+2}=\lambda \widetilde{\mathbf{R}}^{(p)} \boldsymbol{\Theta}_{p}, \quad 1 \leq p \leq M
$$

Matrices $\widetilde{\mathbf{A}}^{(p)}$ to $\widetilde{\mathbf{E}}^{(p)}$ are similar to $\overline{\mathbf{A}}^{(p)}$ to $\overline{\mathbf{E}}^{(p)}$ except that $\Psi$ and $\Omega$ in them have to be replaced by $\bar{\psi}$ and $\bar{\omega}$. The vector $\boldsymbol{\Theta}_{p}$ is a vector of perturbation streamfunction and vorticity values at the location $\tau=\tau_{p}$ similar to $\Phi_{p}$ above. Equation (3.6) can be written in the form of a generalized eigenvalue problem given by

$$
\widetilde{\mathbf{L}} \Theta=\lambda \widetilde{\mathbf{R}} \Theta
$$

where $\widetilde{\mathbf{R}}$ is a diagonal matrix and $\Theta=\left[\Theta_{\mathbf{1}}, \Theta_{\mathbf{2}}, \ldots, \Theta_{\mathbf{M - 1}}, \Theta_{\mathbf{M}}\right]^{T}$. Equation (3.7) is solved for eigenvalues $\lambda$ and eigenvectors $\boldsymbol{\Theta}$ using the software library ARPACK. Interested readers are also referred to the papers by Tuckerman \& Barkley (2000) and Barkley et al. (2008) which give a good discussion of how existing temporal simulation codes may be adapted to solve eigenvalue problems of the type as in (3.7) arising from a primitive variables formulation of the problem.

\subsection{Boundary conditions}

A few of the boundary conditions given in Eqs. (3.2b) and (3.3b) are altered to resolve the flow features and to capture the most unstable modes.

(a) At outflow, the boundary conditions used are

$$
\frac{\partial^{2} \tilde{\psi}}{\partial x^{2}}=-\zeta^{2} \tilde{\psi} \quad \text { and } \quad \frac{\partial^{2} \tilde{\psi}}{\partial y^{2}}-\zeta^{2} \tilde{\psi}+\tilde{\omega}=0
$$

similar to those suggested by Fasel (1976). Here $\zeta$ is a parameter which for the the study of $\mathrm{OE}$ modes was usually set to zero, but for the other modes, a non-zero value (typically 0.1 ) was used. The earlier numerical study on steady flow past a circular cylinder cascade by Gajjar \& Azzam (2004) showed that the above conditions with $\zeta=0$ serve well as non- 


\begin{tabular}{cccccccc}
\hline$x_{\max }$ & $\downarrow$ OBL $\backslash \vec{h}$ & 0.05 & 0.04 & 0.0333 & 0.025 & 0.0167 \\
\hline 216.51 & 19 & 521 & 651 & 781 & 1041 & 1561 \\
300.07 & 21 & 561 & 701 & 841 & 1121 & 1681 \\
403.06 & 23 & 601 & 751 & 901 & 1201 & 1801 \\
527.55 & 25 & 641 & 801 & 961 & 1281 & 1921 \\
675.56 & 27 & 681 & 851 & 1021 & 1361 & 2041 \\
\hline
\end{tabular}

TABLE 1 . Total number of grid points in $\tau$-direction $(M)$ corresponding to various step sizes $(h)$ in each outlet boundary location (OBL in computational domain and $x_{\max }$ in physical domain) that are used in all cases of $W$.

reflecting outlet conditions (with the perturbation quantities replaced by the mean-flow variables).

(b) Using the boundary conditions corresponding to the EE modes $\widetilde{\psi}=0$ and $\widetilde{\omega}=0$ on $A^{\prime} B^{\prime}$ and $E^{\prime} F^{\prime}$ in Fig. 3, we could not find any wake instability even for $R e$ as high as 1000 for the case of $W=5$, and therefore this case is not documented any further below. Unstable modes were found for all the other 3 mode classes as discussed below.

\section{Global stability analysis: results and discussion}

Computations were carried to determine the critical Reynolds number that causes primary wake instability and the associated non-dimensional vortex shedding frequency called the Strouhal number. This is given by $S t_{c}=\frac{\Im\left(\lambda_{c}\right) d}{2 \pi U}$ where $\lambda_{c}$ is the critical eigenvalue which has the largest real part. An attempt was also made to study the effect of blockage ratio between the cylinders and outlet boundary locations on the critical parameters. The gap widths chosen for this study are $W=5,10,20,50$ and 100 corresponding to blockage ratios of $\beta=0.4,0.2,0.04$ and 0.02 respectively. Earlier computations on the steady flow by Gajjar \& Azzam (2004) showed that the location of the upstream boundary, $\tau_{\min }=-7$ (with a corresponding $x_{\min } \approx-90$ ) is sufficient and any further decrease in $\tau_{\min }$ had no significant affect on the results. Therefore, in the current study, $\tau_{\min }=-7$ is chosen. Some studies Behr et al. (1991), Kumar \& Mittal (2006) for example, have shown dependence of the critical parameters on the location of boundaries in the streamwise direction. In order to understand this, we chose $\tau_{\max }=27,25,23,21,19$ (with the corresponding $x_{\max }=675.56,527.55,403.06,300.07$ and 216.51) keeping the $\tau_{\min }$ value fixed. In the steady calculations, Gajjar \& Azzam (2004) used $\tau_{\max }=21$ to ensure that the eddy was contained in the computational domain. So, we chose a slightly lower value than this minimum value $\left(\tau_{\max }=19\right)$ to find if this is sufficient for computations. The maximum value of the outlet boundary location (OBL) is set to 27 as any value greater than this was found to be computationally expensive. OBL is same as $\tau_{\max }$. But this acronym will be used hereafter for convenience.

Tables 1 and 2 show the values of $M$ and $N$ typically used for our computations. The maximum value of $h$ which is the step size in $\tau$-direction is chosen to be 0.05 as any value higher than this was not found to be good enough to capture the flow features. The minimum value of $h$ is chosen as 0.0167 as the unsteady calculations for any value less than this is computationally expensive especially when $W$ is either 50 or 100 and for larger values of the OBL. 


\begin{tabular}{ccccc}
\hline$W$ & $\beta$ & \multicolumn{3}{c}{$N$} \\
\hline 5 & 0.4 & 16 & 32 & 64 \\
10 & 0.2 & 16 & 32 & 64 \\
20 & 0.1 & 32 & 64 & 81 \\
50 & 0.04 & 64 & 81 & 101 \\
100 & 0.02 & 64 & 81 & 101 \\
\hline
\end{tabular}

TABLE 2. Total number of grid points in $\eta$-direction $(N)$ that are used in different cases of $W$.

\begin{tabular}{|c|c|c|c|c|c|}
\hline$W$ & $\beta$ & $R e_{c}$ & $S t_{c}$ & $x_{p}$ & $\tilde{u}_{p}$ \\
\hline 5 & 0.4 & 50.8 & 0.2952 & 14.1091 & 0.5407 \\
\hline 10 & 0.2 & 49.6 & 0.1726 & 37.9521 & 0.2414 \\
\hline 20 & 0.1 & 49.8 & 0.1358 & 56.7349 & 0.1334 \\
\hline 50 & 0.04 & 48.9 & 0.1211 & 115.7791 & 0.0149 \\
\hline 100 & 0.02 & 48.8 & 0.1181 & 121.0447 & $1.165710^{-4}$ \\
\hline
\end{tabular}

TABLE 3. Critical values at different blockage ratios for the OE modes. Here $x_{p}$ is the location for the maximum value (denoted by $\tilde{u}_{p}$ ) of the centerline perturbation velocity $\left|u_{1}\right|$. The outlet boundary was set at $\tau_{\max }=21$ corresponding to $x_{\max }=300.07$

\subsection{Results for the OE modes}

We first present results for the odd-even modes (as defined in section 3 ). The blockage is defined as $\beta=\frac{2}{W}$ and the respective critical values corresponding to the OE modes are tabulated in Table 3 where we show the critical values of the Reynolds number $R e_{c}$ and the critical Strouhal frequencies $S t_{c}$ for some of the blockage ratios studied at the onset of instability, taken from the finest grid data. Figure 4(a) clearly indicates that with increase in blockage, there is an increase in $S t_{c}$. As the blockage value increases, $R e_{c}$ increases and then decreases and again increases as shown in Fig. 4(b). The one other noticeable effect of blockage is that the maximum centerline perturbation velocities in the wake region increase with increasing blockage from $O\left(10^{-4}\right)$ for $\beta=.02$ to approximately 0.6 for $\beta=0.4$, see Table 3 .

In figures 5-9 we have shown contour plots of the streamfunction and vorticity for the basic flow, together with contour plots of the real and imaginary parts of the eigenfunctions at the critical values. For clarity, the eigenfunction plots for blockage ratios $\beta<0.2$, are shown for only a partial domain in both $x$ and $y$, and not the full domain used for the computations. The basic flow shows a reversed flow eddy with the reattachment point between $x=5$ and $x=7.5$ for all the blockage ratios studied, and the reattachment point is closer to the cylinder with increased blockage.

Figure 10 shows the computed eigenvalues for the case when $W=100$ for one particular grid. The eigenvalues crossing the imaginary axis lead to a Hopf bifurcation and are well separated from the others. Interestingly the spectrum also shows another low frequency discrete pair with $\Re(\lambda)=-0.08$ approximately. Although we have not carried 

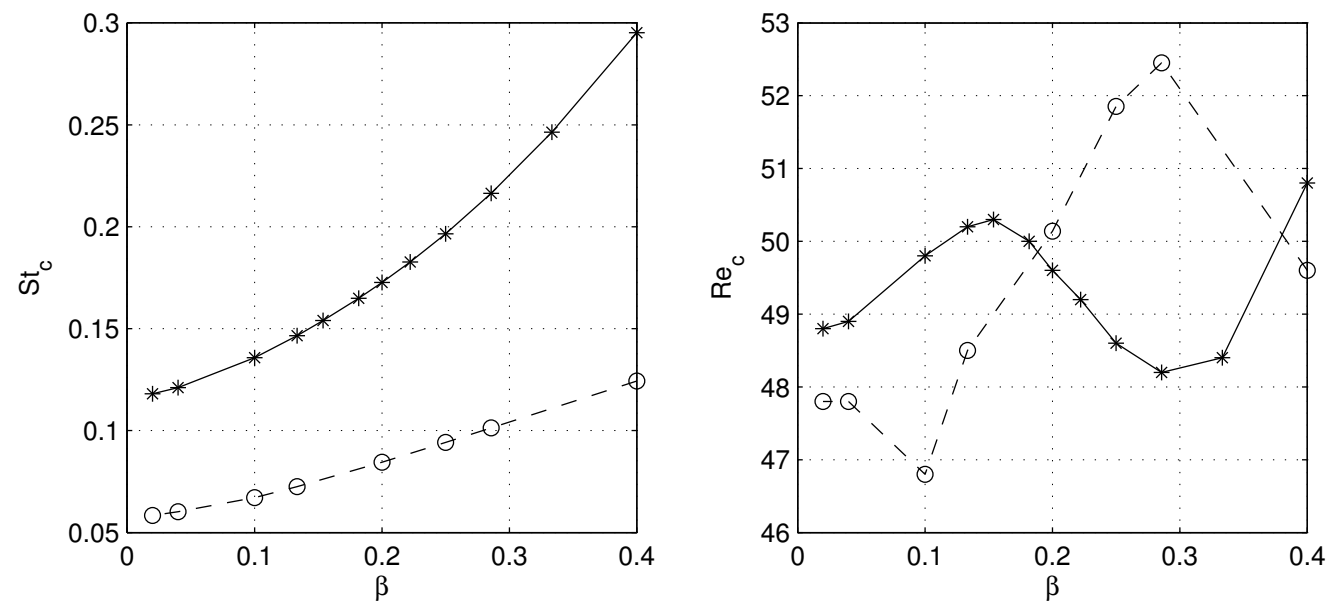

FiguRE 4. Graphs of the (a) critical Strouhal number $S t_{c}$ and (b) critical Reynolds number $R e_{c}$ versus blockage ratio $\beta$ for the OE modes (solid line and asterix) and OO modes (dashed line and circles).

\begin{tabular}{ccc}
\hline$R e$ & $\lambda_{1}$ & $\lambda_{2}$ \\
\hline 100 & $0.070589 \pm 0.42866 i$ & - \\
125 & $0.0874 \pm 0.4194 i$ & - \\
150 & $0.098684 \pm 0.40867 i$ & $0.024788 \pm 0.44014 i$ \\
175 & $0.10668 \pm 0.39827 i$ & $0.039963 \pm 0.43068 i$ \\
185 & $0.10923 \pm 0.39437 i$ & $0.044857 \pm 0.42664 i$ \\
200 & $0.11254 \pm 0.38883 i$ & $0.051213 \pm 0.42058 i$ \\
\hline
\end{tabular}

TABLE 4. Unstable eigenvalues $\lambda_{1}, \lambda_{2}$ at higher values of the Reynolds number for the case $W=20, \beta=0.1$ with $M=601, N=32$, OE modes.

out extensive computations to find additional unstable OE modes at higher Reynolds numbers, these are present. For instance, for $W=20$, in Table 4, additional unstable eigenvalues for Reynolds numbers between 125 and 200 are shown, and it is clear that a second unstable pair becomes unstable between $R e=125$ and 150 . The same conclusion also applies to the $W=50, \beta=0.04$ case. This can be seen clearly also in Fig. 11 where a plot of the spectrum is shown for $W=20, \beta=0.1$, and $W=50, \beta=0.04$ is shown.

The eigenfunctions are all normalised so that the maximum absolute value of the streamfunction eigenvector is unity. Only the details near the cylinder are shown for most of the cases. What is noticeable is that all the eigenvector plots show a wavepacket behaviour and the flow near the cylinder appears periodic after about 2.5 cylinder diameters and persists for some considerable distance downstream. The vorticity eigenvectors have a staggered double humped shape with negative and positive values, and with increased blockage, the more intense are the peak values. The maximum and minimum values of the vorticity eigenvectors are located on the $y=0$ line. For the high blockage ratios the location of the secondary peaks is between $y=1$ and $y=2$ and this line moves further away for decreasing $\beta$. 
(a)
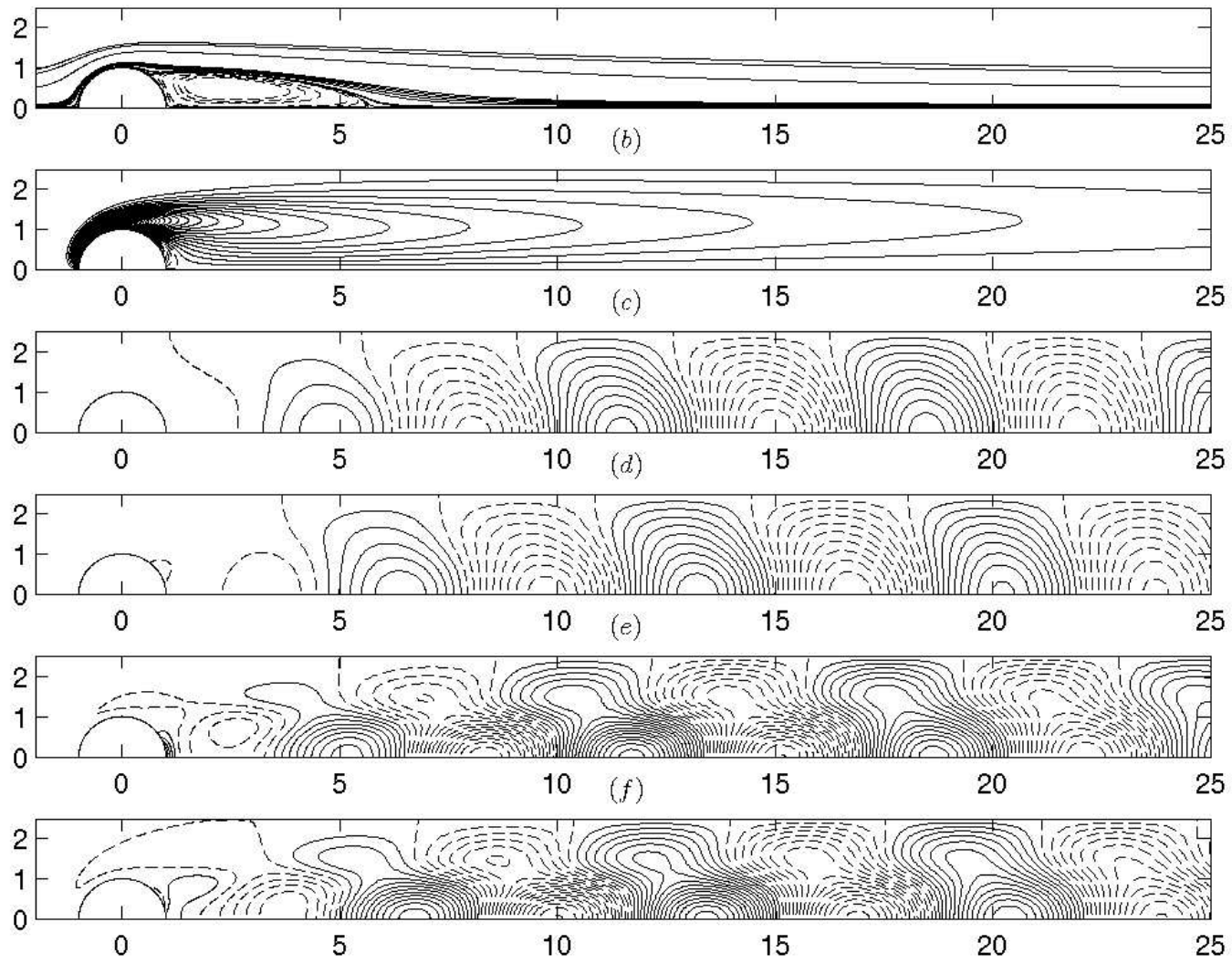

FIGURE 5. Contours of (a) streamfunction (b) vorticity (c) real part of stream function eigenvector, (d) imaginary part of stream function eigenvector, (e) real part of vorticity eigenvector and (f) imaginary part of vorticity eigenvector for OE modes at $R e_{c}=50.8$ for $W=5, \beta=0.4$, $(M=1681, N=64$ and $\mathrm{OBL}=21)$. The dashed contours denote negative values.

The details of the eigenfunctions for the cases when $W=50$ and $W=100$, see Figures 8,9 are almost identical in the near wake region and also far downstream. This can be seen clearly in Fig. 13 where we plotted the streamfunction on the line $y=0$ for both cases. With increased blockage, the location of the maximum of the streamfunction eigenfunction moves closer to the cylinder and decays faster. This is shown clearly in Fig. 14, as well as in the tabulated data for the centerline velocities in Table 3. In Fig. 15 we have plotted the function $\tilde{\omega}_{m}=\max [|\tilde{\omega}(x, y)|]$ for various blockage ratios. This shows that for increased blockage the maximum of the envelope of the vorticity eigenfunctions also moves closer to the cylinder. In addition for small blockages $\beta<0.2$ the peaks coincide at approximately the same location. An anonymous referee has suggested that this may have some connection with the findings of Goujan-Durand et al. (1994). The slight oscillations present in the plots for $W=100, \beta=0.02$ are because of the coarser grid used. 
(a)
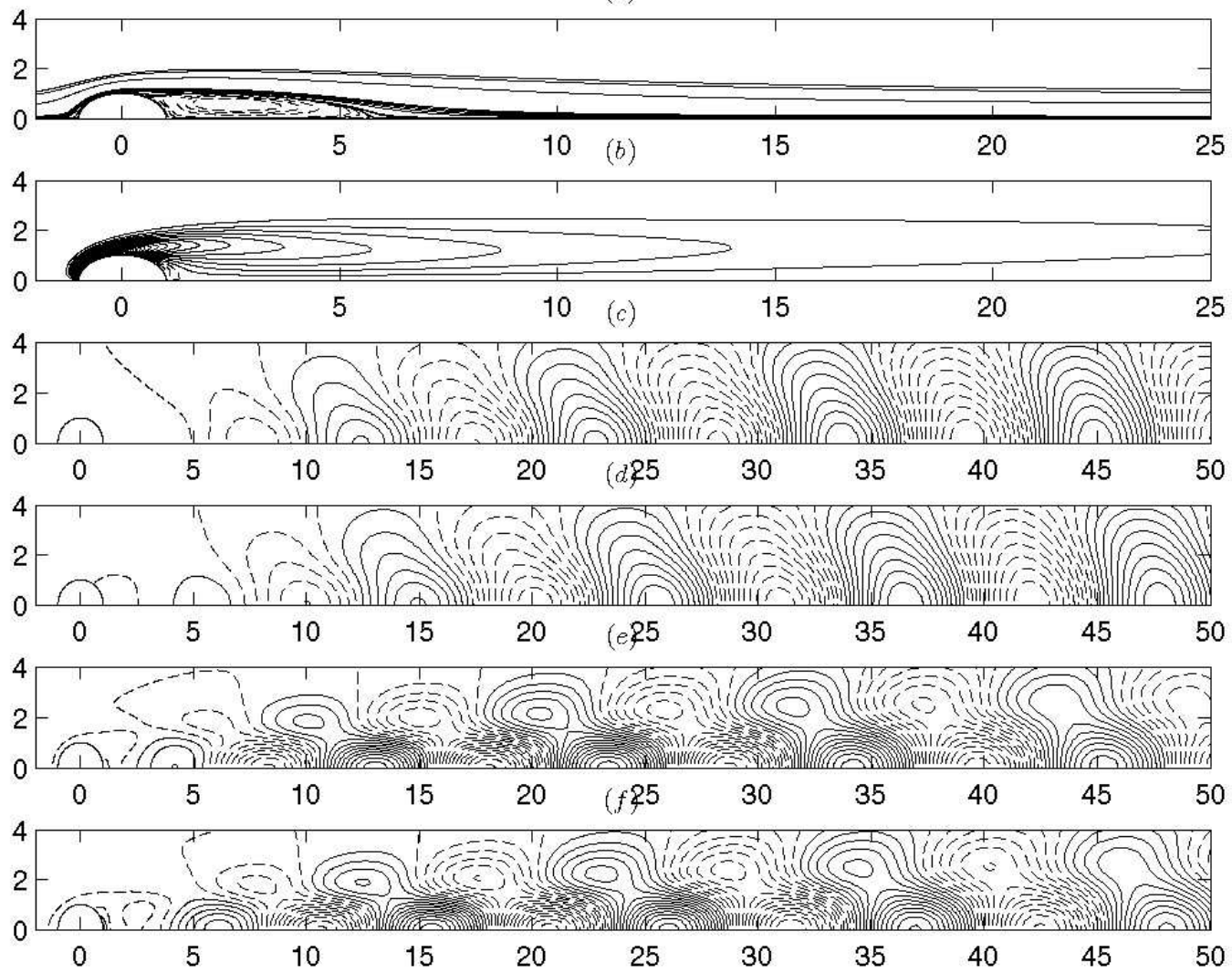

FiguRE 6. Contours of (a) streamfunction (b) vorticity (c) real part of stream function eigenvector, (d) imaginary part of stream function eigenvector, (e) real part of vorticity eigenvector and (f) imaginary part of vorticity eigenvector for OE modes at $R e_{c}=49.6$ for $W=10, \beta=0.2$, $(M=1681, N=64$ and $\mathrm{OBL}=21)$.

\subsection{Results for $O O$ modes}

In Table 5 the values for the critical parameters for the onset of instability of the OO mode class are given with the instability again taking place as a Hopf bifurcation. The critical Reynolds number for the onset of instability does not change greatly with change in blockage ratio and the values are not too dissimilar to those for the OE modes. The variation of $R e_{c}$ is also non-monotonic with change in blockage ratio, see figure 4 . The OO modes have much lower Strouhal frequencies than for the OE modes and increase in value with increasing blockage ratio. For low blockages, the OO mode is the first to become unstable as compared to the OE mode as can be seen in figure 4.

The spectrum for the OO modes computed for $W=50$ is shown in Fig. 12, and shows a pair of well separated unstable eigenvalues. Taking $\zeta$ non-zero in the outer boundary condition it was found that for some blockage ratios it was not possible to pick up the unstable eigenvalues. Moreover, the eigenfunctions (with $\zeta=0$ ) for the least stable modes showed growth at the downstream boundaries and these were not grid independent. The 
$(a)$
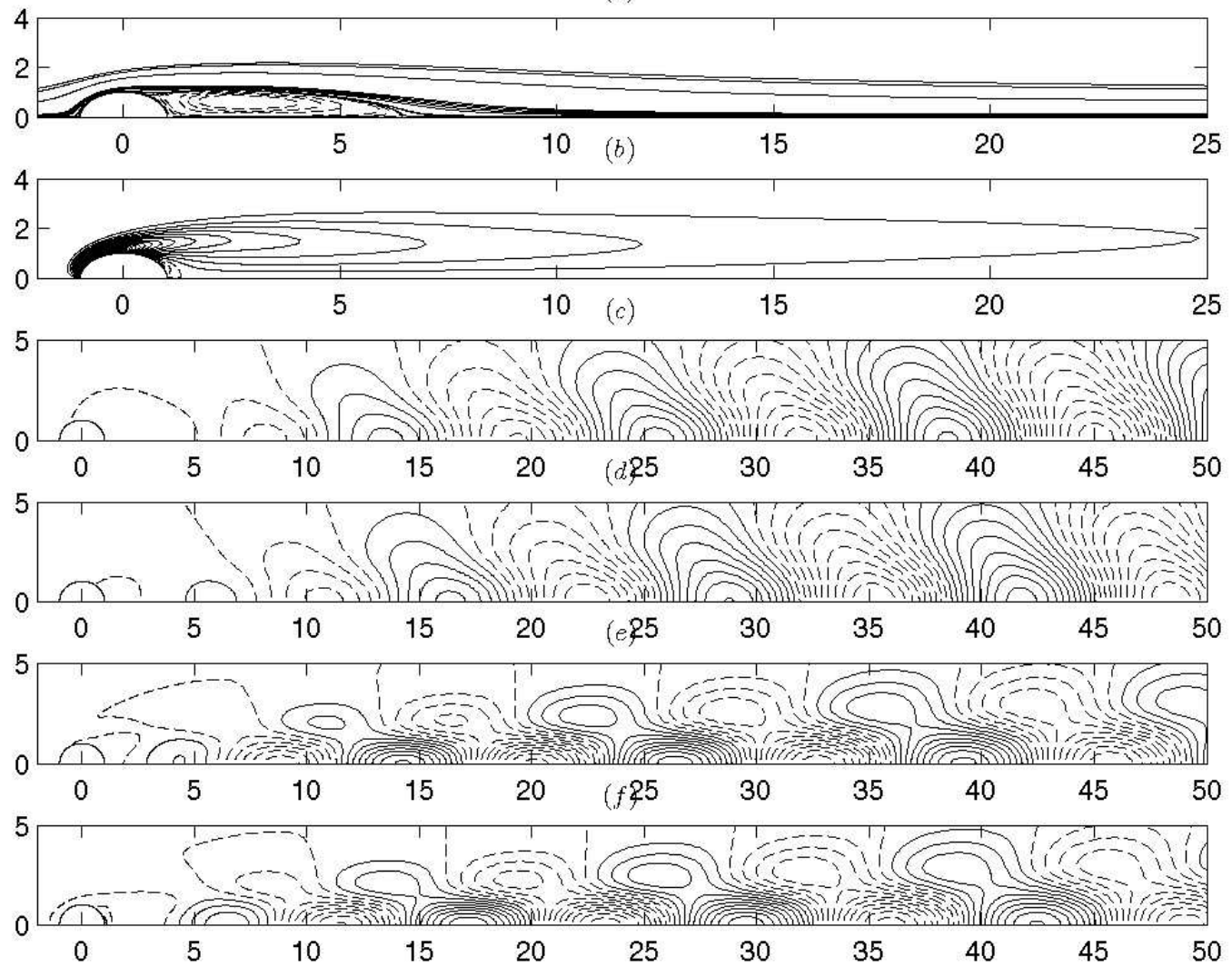

FIGURE 7. Contours of (a) streamfunction (b) vorticity (c) real part of stream function eigenvector, (d) imaginary part of stream function eigenvector, (e) real part of vorticity eigenvector and (f) imaginary part of vorticity eigenvector for $\mathrm{OE}$ modes at $R e_{c}=49.8$ for $W=20, \beta=0.1$, $(M=1681, N=81$ and $\mathrm{OBL}=21)$.

values for the critical values shown in Table 5 were checked by varying various parameters and found to be fairly robust.

Eigenfunctions for the OO modes are shown in figures 17-19 for blockage ratios $\beta=$ $0.02,0.1$ and 0.4 . The real and imaginary parts of the eigenfunctions look similar, offset by a small phase. For large blockages the eigenfunctions peaks are staggered along the cylinder centreline $(y=0)$ as compared to the peaks on the mid-lane between the cylinders. The shape of the eigenfunctions on the wake centerline $(y=0)$ shows a typical wave-packet behaviour as can be seen in figure 16. The disturbances extend some considerable distance downstream and eventually decay. For larger blockage ratios the decay is faster than that for the smaller blockage ratios. In figure 16 the real parts of the centerline perturbation streamfunction are compared for $W=50$ and $W=100$ and shows that the results for $W=50$ capture the dominant features for the large gap-width case. 
(a)
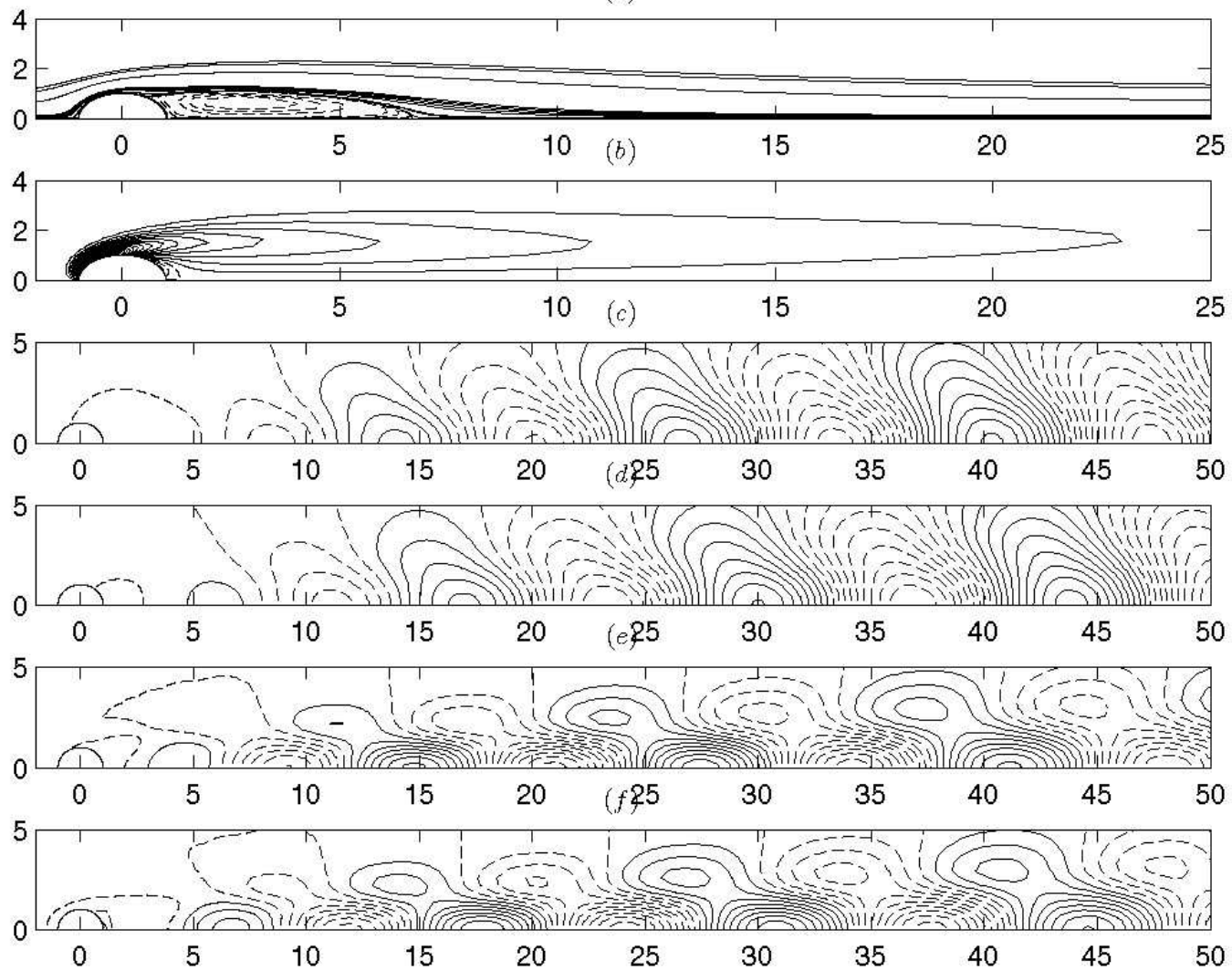

Figure 8. Contours of (a) streamfunction (b) vorticity (c) real part of stream function eigenvector (d) imaginary part of stream function eigenvector (e) real part of vorticity eigenvector and (f) imaginary part of vorticity eigenvector for $\mathrm{OE}$ modes at $R e_{c}=48.9$ for $W=50, \beta=0.04$, $(M=1681, N=101$ and $\mathrm{OBL}=21)$.

\begin{tabular}{|c|c|c|c|c|c|}
\hline$W$ & $\beta$ & $R e_{c}$ & $\Re(\lambda)$ & $\Im(\lambda)$ & $S t_{c}$ \\
\hline 5 & 0.4 & 49.6 & $2.310^{-4}$ & \pm 0.7810 & 0.12431 \\
\hline 10 & 0.2 & 50.14 & 3. $10^{-4}$ & \pm 0.5306 & 0.08443 \\
\hline 20 & 0.1 & 46.8 & $-2.310^{-4}$ & \pm 0.4218 & 0.06731 \\
\hline 50 & 0.04 & 47 & $-8.210^{-5}$ & \pm 0.3783 & 0.06044 \\
\hline 100 & 0.02 & 47.8 & $6.410^{-5}$ & \pm 0.3696 & 0.05883 \\
\hline
\end{tabular}

TABLE 5. Critical values for the onset of instability for the OO modes. The results are for a grid size with $N=64, M=1021$ points. 
$(a)$
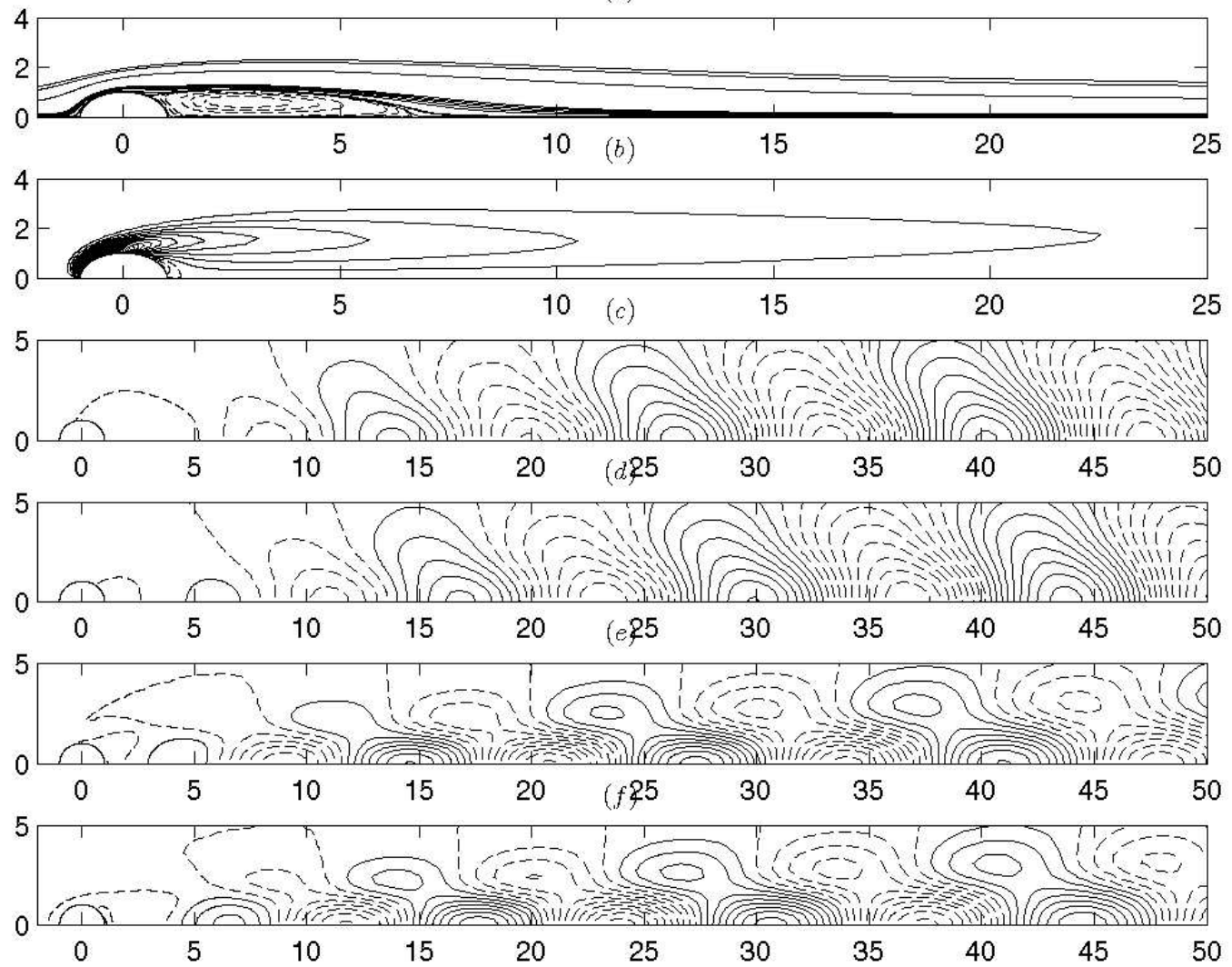

FIgURE 9. Contours of (a) stream function, (b) vorticity, (c) real part of stream function eigenvector, (d) imaginary part of stream function eigenvector, (e) real part of vorticity eigenvector and (f) imaginary part of vorticity eigenvector for OE modes at $R e_{c}=48.7$ for $W=100, \beta=0.02$, $(M=1681, N=101$ and $\mathrm{OBL}=21)$.

\subsection{Results for EO modes}

Critical values for the onset of instability for the EO modes are given in Table 6. It can be seen that for this mode class, the instability is via a pitchfork bifurcation. In all cases of the blockage ratios computed, the critical Reynolds numbers are much higher values than those for the $\mathrm{OE}$, and $\mathrm{OO}$ modes, with $R e_{c}$ increasing with decreasing blockage ratios.

Typical eigenfunctions for the EO modes are shown in figure 20 for the case $W=5$. The eigenfunction peaks close to the cylinder and decays rapidly within 50 cylinder diameters. The vorticity eigenfunction also shows the positive and negative peaks on either side of the separated streamline of the steady flow. The imaginary part of the vorticity eigenfunction should be zero but has a small non-zero value arising from numerical error. If the value for $\zeta$ in the boundary condition at the outlet is zero, then the numerical error increases significantly downstream. 
(a)

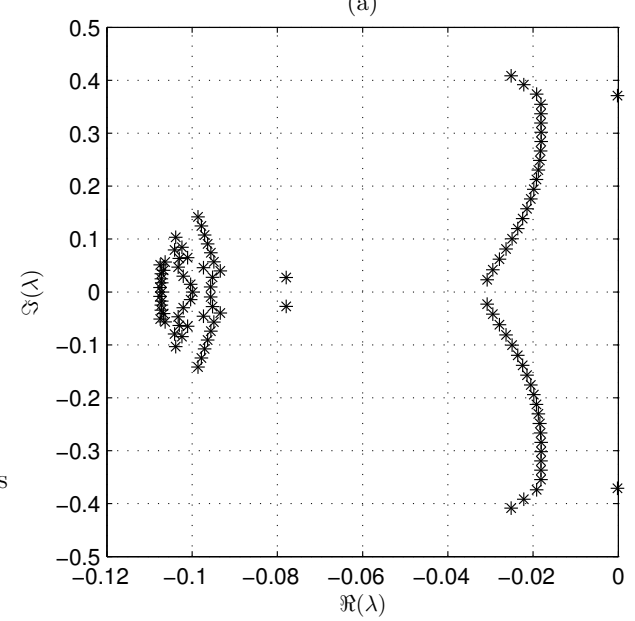

(b)

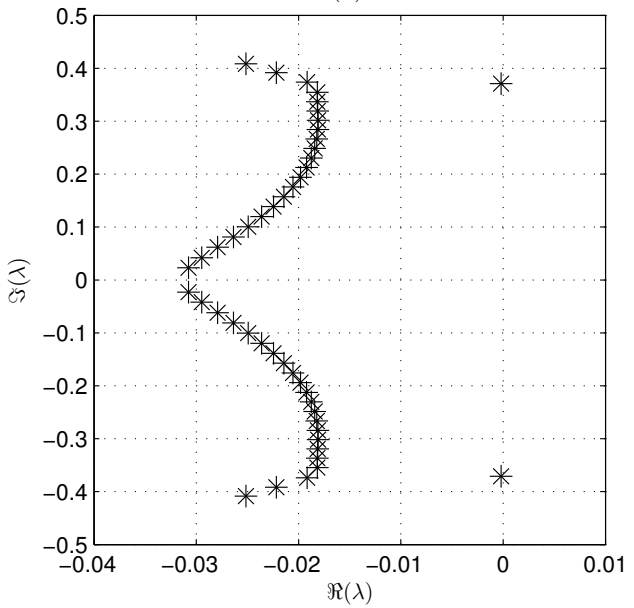

FIgURE 10. Plots showing (a) 100 eigenvalues and (b) few eigenvalues near the imaginary axis for $\mathrm{OE}$ modes at $R e_{c}=48.7$ for $W=100, \beta=0.02,(M=1681, N=101$ and $\mathrm{OBL}=21)$.

(a)

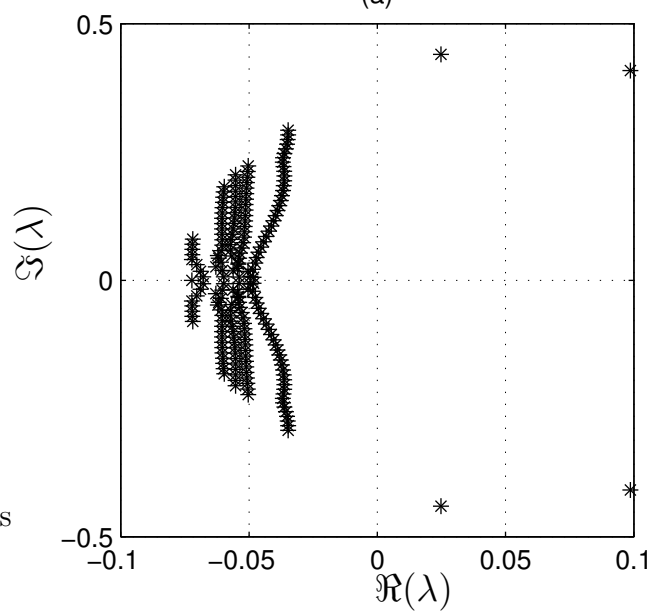

(b)

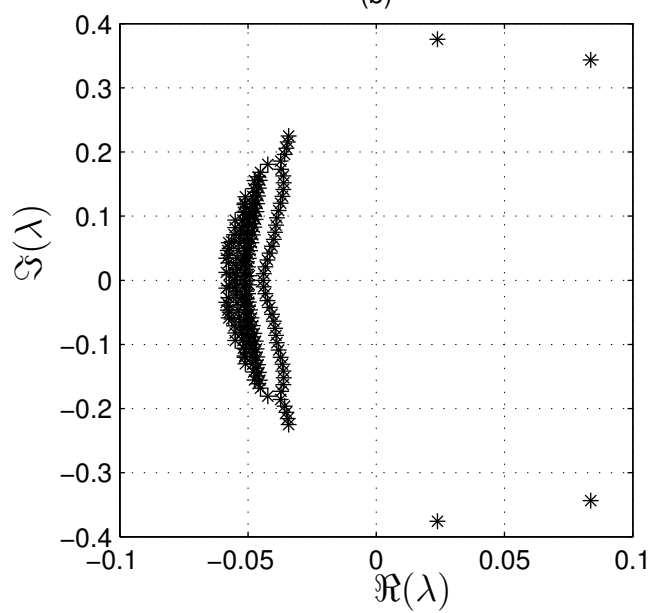

FiguRE 11. Spectrum for (a) $W=20, \beta=.1$ and (b) $W=50, \beta=0.04$ with $R e=150$ for the OE modes.

\begin{tabular}{ccccc}
\hline$W$ & $\beta$ & $R e_{c}$ & $\Re(\lambda)$ & $\Im(\lambda)$ \\
\hline 5 & 0.4 & 68.7 & $5.10^{-5}$ & 0.0 \\
10 & 0.2 & 139.25 & $-2.10^{-5}$ & 0.0 \\
20 & 0.1 & 212.025 & $2.10^{-5}$ & 0.0 \\
50 & 0.04 & 334.536 & $7.10^{-8}$ & 0.0 \\
\hline
\end{tabular}

TABLE 6. Critical values for the onset of instability for the EO modes. The results are for a grid size with $N=64, M=641$ points. 
(a)

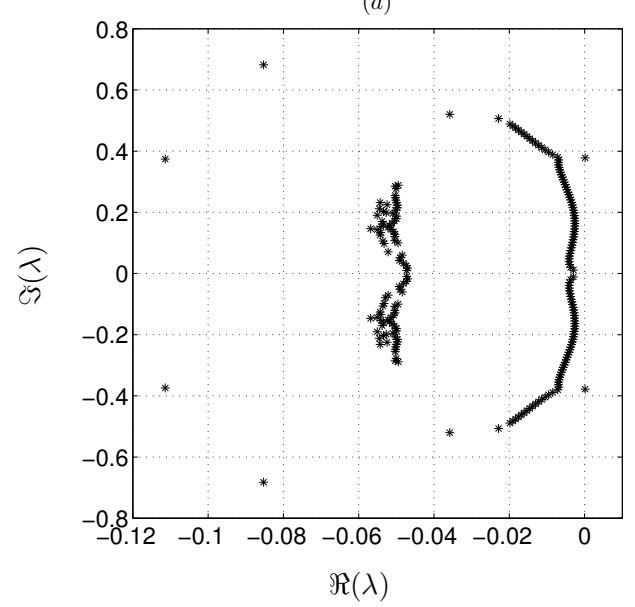

(b)

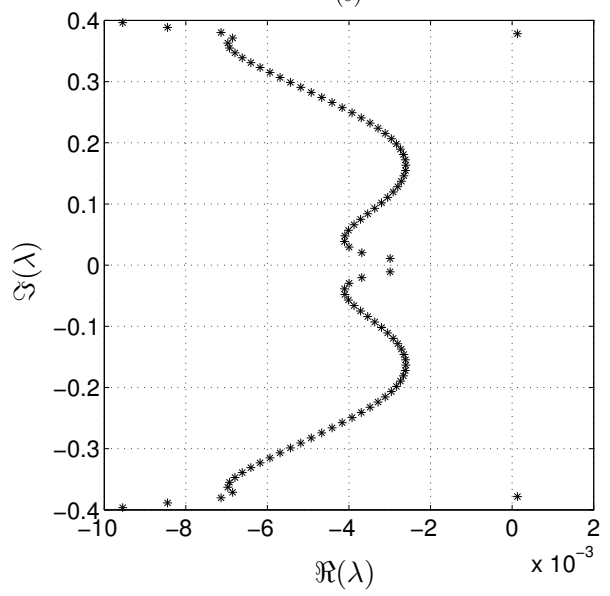

FiguRE 12. Spectrum for (a) $W=50, R e=47.1$ (b) a closeup view for the OO modes, with $N=64, M=1021$.

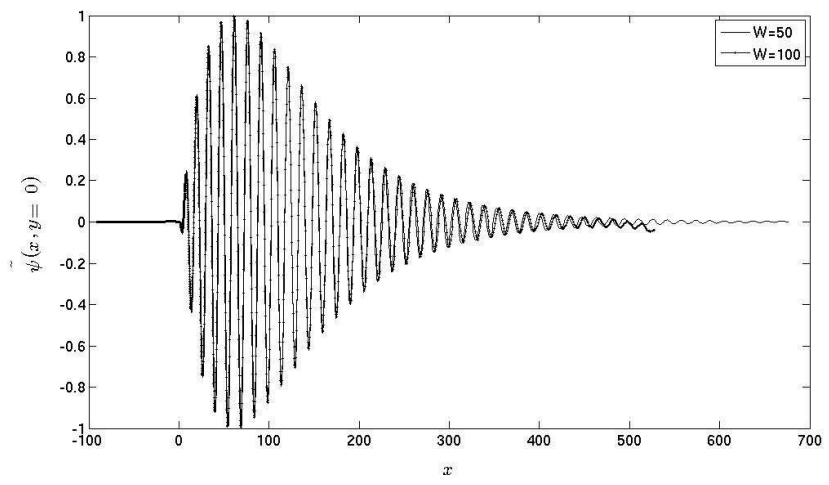

Figure 13. Comparison of perturbation streamfunction $\tilde{\psi}(x, y=0)$ for the cases $W=50,100,(\beta=0.04,0.02)$, OE modes.

\subsection{Effect of grid size and downstream boundary location on critical values.}

The critical values cited in Tables 3, 5 were found to be grid-independent for the cases $W=5,10,20,50$ provided the OBL was located sufficiently far enough downstream. The values range from $O B L=19$ for the case $W=5$ to $O B L=25$ for the case $W=50$. At these locations the perturbations are of sufficiently small magnitude to not affect the solution. On the other hand, the computations for the $W=100$ case proved particularly sensitive to the choice of the OBL as well as the number of basis vectors in the Arnoldi iterations, especially for the OE modes.

The details of the typical parameters used to compute $R e_{c}$ and $S t_{c}$ for $W=100$ are tabulated in Table 8. As with any computations using ARPACK, the convergence 


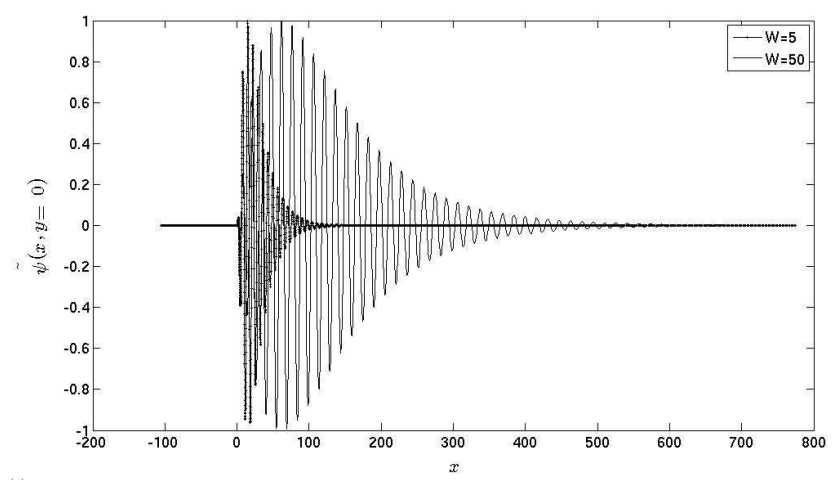

FiguRE 14. Comparison of perturbation streamfunction $\tilde{\psi}(x, y=0)$ for the cases $W=5,50,(\beta=0.4,0.04)$, OE modes.

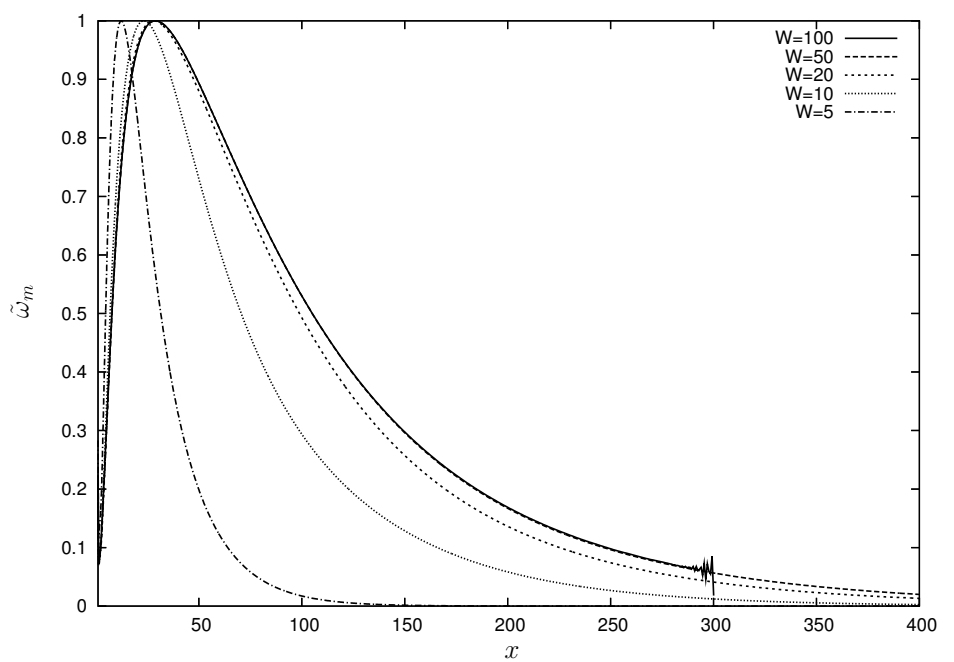

Figure 15. A plot of the maximum of the envelope of the vorticity eigenfunction $\tilde{\omega}_{m}$ versus $x$ for various blockage ratios, OE modes.

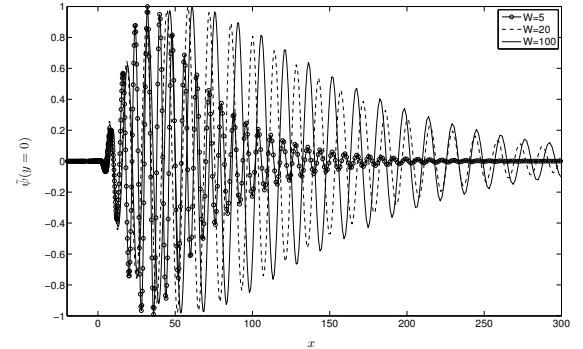

(a)

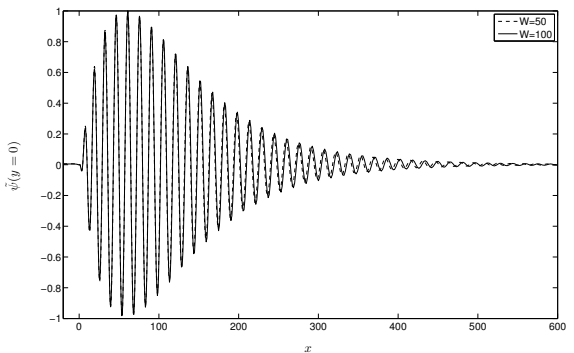

(b)

FiguRE 16. (a) A plot of the real part of the disturbance eigenfunction $\tilde{\psi}(x, y=0)$ versus $x$ for various blockage ratios, OO modes and (b) a comparison of the same for $W=50$ and $W=100$. 
(a)
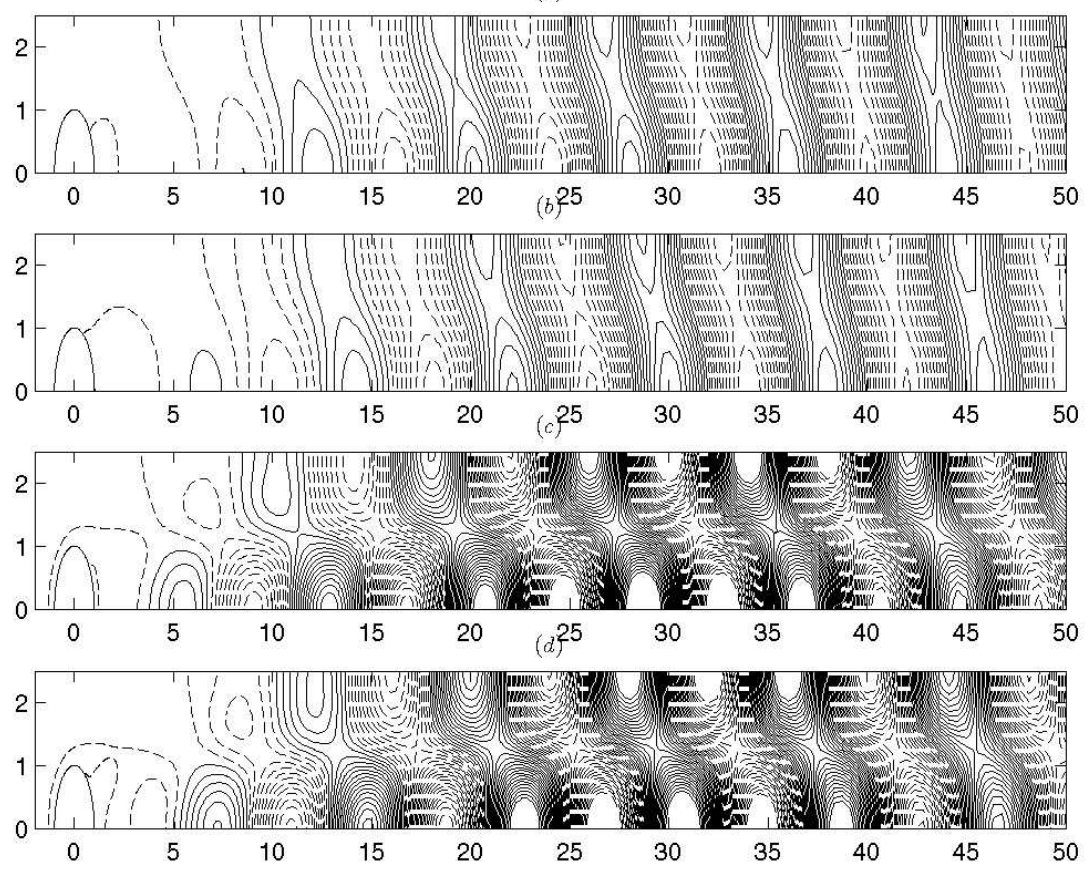

Figure 17. Contours of (a) real part of stream function eigenvector, (b) imaginary part of stream function eigenvector, (c) real part of vorticity eigenvector and (d) imaginary part of vorticity eigenvector for $\mathrm{OO}$ modes at $R e_{c}=49.6$ for $W=5, \beta=0.4,(M=1021, N=64)$.

properties depend critically on the choice of the number of basis vectors, converged eigenvalues, as well as the shift parameters which are used.

Whereas the critical values for the Reynolds number and Strouhal frequencies show only a small variation with respect to the grid parameters used, with the parameter $\zeta=0$ taken for the downstream boundary condition, the location of the OBL for $W=100$ had a major effect on the eigenfunction. This is seen clearly in calculating the streamwise velocity, $u_{1}=\Re\left(\widetilde{\psi}_{y}\right)$, corresponding to finest grid size for $\mathrm{OBL}=21$ and 25 . The centerline $(y=V / 2)$ velocity profiles are shown in Fig. 21 . It is noted that for $\mathrm{OBL}=21$ the wave packets form just behind the cylinder and as they approach the outlet boundary, the oscillations drift away from their mean fluctuation level. However, the wave packet of $u_{1}$ corresponding to $\mathrm{OBL}=25$ behind the cylinder has gradually decayed but at the downstream boundary, its magnitude has again increased. In Fig. 22 we present centerline velocities for two different OBL's for the case when $W=10$. This shows grid independence as compared to the $W=100$ case primarily because the eigenfunction has decayed sufficiently for the enforcement of the boundary condition to be feasible. This is also seen clearly in the contour plots for the eigenfunctions in Fig. 23 where we have shown the eigenfunctions over the full extended domain with the $\mathrm{OBL}=21$. Even though the outer boundary is set at 150 diameters from the cylinder the effect on the streamfunction and vorticity eigenfunctions is considerable near the outer boundary. In Fig 24 we have shown similar contour plots for the $W=100$ eigenfunctions when the outlet boundary is set to a much larger value $(\mathrm{OBL}=25)$. Here the streamfunction eigenvectors are not affected by 
(a)
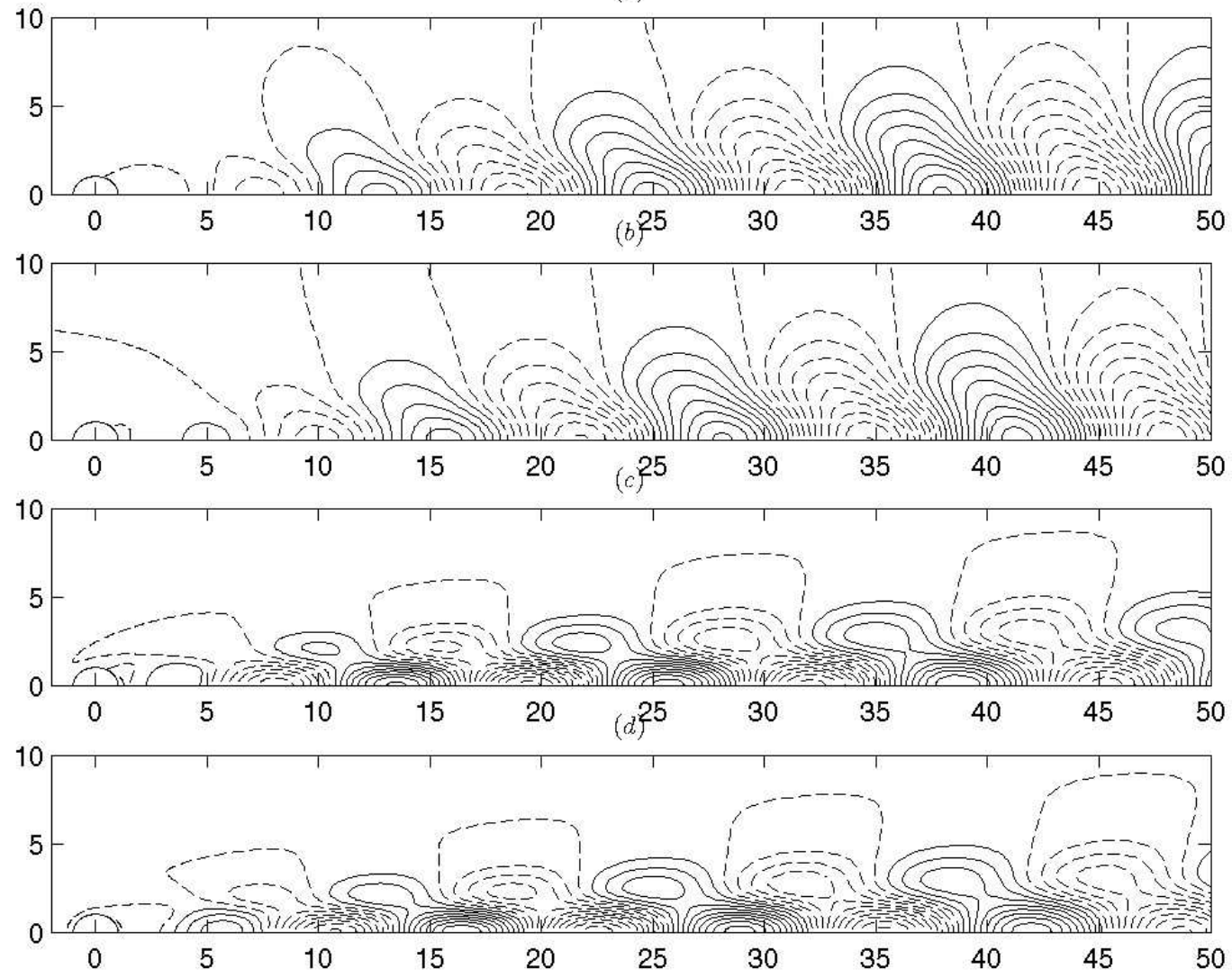

Figure 18. Contours of (a) real part of stream function eigenvector, (b) imaginary part of stream function eigenvector, (c) real part of vorticity eigenvector and (d) imaginary part of vorticity eigenvector for $\mathrm{OO}$ modes at $R e_{c}=47$ for $W=20, \beta=0.1,(M=1021, N=64)$.

the downstream boundary, but the vorticity eigenvectors still show some distortion close to the downstream boundary.

For the EO modes, choosing a non-zero value for $\zeta$ led to growth of the eigenfunction at the downstream boundary, with the details in the region closest to the cylinder $(x<250)$ remaining largely unchanged. In addition for the EO modes, since the bifurcation is via a pitchfork bifurcation, the computed numerical approximation to the (zero) imaginary part of the eigenfunction manifested as a significant numerical (point to point) oscillation with a zero mean.

The computation of the OO modes required a careful choice of the shift parameter using in ARPACK. In many instances, taking this to be zero or too small failed to pick up the unstable eigenvalues. Taking a zero value for $\zeta$ in the outlet boundary condition also caused difficulties with the computed eigenfunction growing at the downstream boundary, and in some cases this also did not pick up most unstable eigenvalue. 
(a)
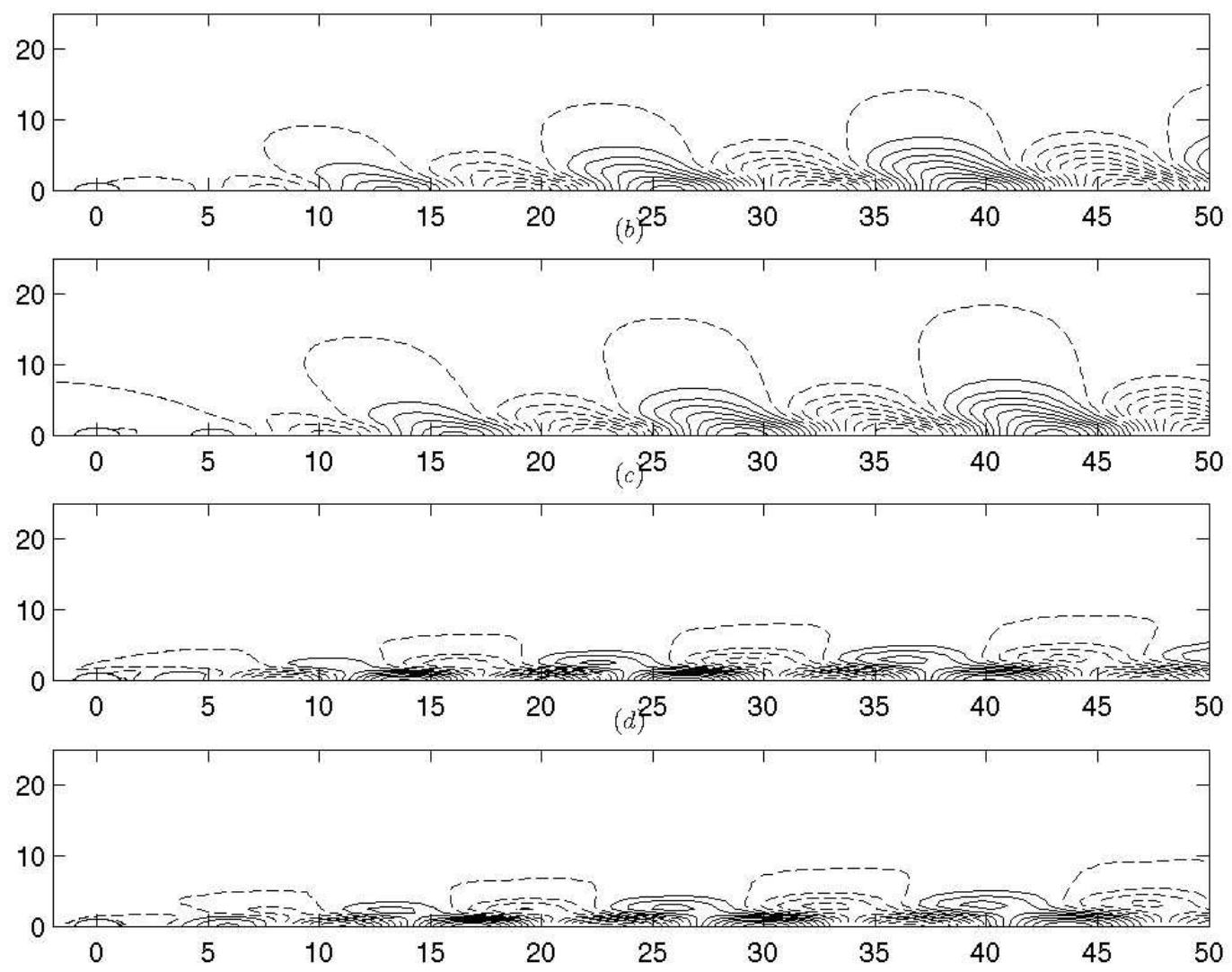

FIGURE 19. Contours of (a) real part of stream function eigenvector, (b) imaginary part of stream function eigenvector, (c) real part of vorticity eigenvector and (d) imaginary part of vorticity eigenvector for $\mathrm{OO}$ modes at $R e_{c}=47.8$ for $W=100, \beta=0.02,(M=1021, N=64)$. Note that only the partial domain is shown.

\begin{tabular}{cccccccccccccc}
\hline \multicolumn{2}{c}{$\mathrm{OBL}=25, N=64$} & \multicolumn{2}{c}{$\mathrm{OBL}=23, N=64$} & \multicolumn{2}{c}{$\mathrm{OBL}=21, N=101$} & \multicolumn{2}{c}{$\mathrm{OBL}=19, N=101$} \\
\hline$M$ & $R e_{c}$ & $S t_{c}$ & $M$ & $R e_{c}$ & $S t_{c}$ & $M$ & $R e_{c}$ & $S t_{c}$ & $M$ & $R e_{c}$ & $S t_{c}$ \\
\hline 641 & 48.8 & 0.1181 & 601 & 48.7 & 0.1180 & 561 & 48.8 & 0.1181 & 521 & 48.5 & 0.1181 \\
801 & 48.8 & 0.1181 & 751 & 48.7 & 0.1181 & 701 & 48.8 & 0.1181 & 651 & 48.5 & 0.1181 \\
961 & 48.8 & 0.1181 & 901 & 48.7 & 0.1181 & 841 & 48.7 & 0.1181 & 781 & 48.5 & 0.1181 \\
1281 & 48.8 & 0.1181 & 1201 & 48.7 & 0.1181 & 1121 & 48.7 & 0.1181 & 1041 & 48.5 & 0.1181 \\
1921 & 48.8 & 0.1181 & 1801 & 48.7 & 0.1181 & 1681 & 48.7 & 0.1181 & 1561 & 48.5 & 0.1181 \\
\hline
\end{tabular}

TABLE 7 . Numerical results for varying outlet locations $(W=100, \beta=0.02)$, OE modes. 


\begin{tabular}{ccccccc}
\hline \multirow{2}{*}{ OBL } & \multicolumn{3}{c}{ Coarse grid } & \multicolumn{3}{c}{ Fine grid } \\
\cline { 2 - 7 } & NEV & NCV & Grid size & NEV & NCV & Grid size \\
\hline 19 & 100 & 2500 & $64 \times 521$ & 100 & 2500 & $101 \times 1561$ \\
21 & 100 & 1000 & $64 \times 561$ & 100 & 1000 & $101 \times 1681$ \\
23 & 301 & 2000 & $101 \times 601$ & 375 & 2000 & $101 \times 1801$ \\
25 & 750 & 2250 & $64 \times 641$ & 801 & 2250 & $64 \times 1921$ \\
\hline
\end{tabular}

TABle 8. Computational details for $W=100, \beta=0.02$, ARPACK shift parameter $(\sigma=1.0)$.

\begin{tabular}{lcccc}
\hline Researcher(s) & $R e_{c}$ & $S t_{c}$ & Method & Grid points/Mesh size \\
\hline Kovásznay (1949) & 40 & - & Experiments & - \\
Coutanceau \& Bouard (1977) & $34-43$ & - & Experiments & - \\
Nishioka \& Sato (1978) & 48 & 0.12 & Experiments & - \\
et al. Gresho et al. (1984) & 50 & 0.14 & FEM & 1852 \\
Jackson (1987) & 46.184 & 0.13804 & FEM & Extrapolation \\
Zebib (1987) & 40 & 0.12 & SM & - \\
Williamson (1989) & 47.9 & 0.1220 & Experiments & - \\
Yang \& Zebib (1989) & $40-45$ & 0.115 & SM & - \\
Morzyński \& Thiele (1991) & 46.23 & 0.1345 & FDM & - \\
Strykowski \& Hanneman (1991) & 42 & - & FDGM & Extrapolation \\
Noack \& Eckelmann (1994) & 54 & 0.149 & GM & 63 \\
et al. Chen et al. (1995) & 47.9 & 0.138 & FEM & - \\
Henderson (1995) & $46 \pm 1$ & - & SM & 202 \\
Ding \& Kawahara (1999) & 46.389 & 0.126 & FEM & - \\
Morzyński et al. (1999) & 47 & 0.1320 & FEM & 15838 \\
Ding (2003) & 47.23 & - & FEM & - \\
Sahin \& Owens (2004) & 46.74 & 0.1167 & FVM & Extrapolation \\
Cadou et al. (2006) & 45.61 & 0.163 & FEM & 6400 d.o.f \\
Kumar \& Mittal (2006) & 47.336 & 0.1168 & FEM & 34324 \\
This study & 48.8 & 0.1181 & FDM \& CCM & $64 \times 1921$ \\
& & & &
\end{tabular}

TABLE 9. Comparison of critical values with literature, OE modes.

Description of acronyms used in Table 9:

FEM - Finite Element Method.

FDGM - Finite Difference Galerkin Method.

GM - Galerkin Method.

SM - Spectral Method.

FVM - Finite Volume Method.

FDM - Finite Difference Method.

CCM - Chebyshev Collocation Method.

d.o.f - degrees of freedom. 
(a)
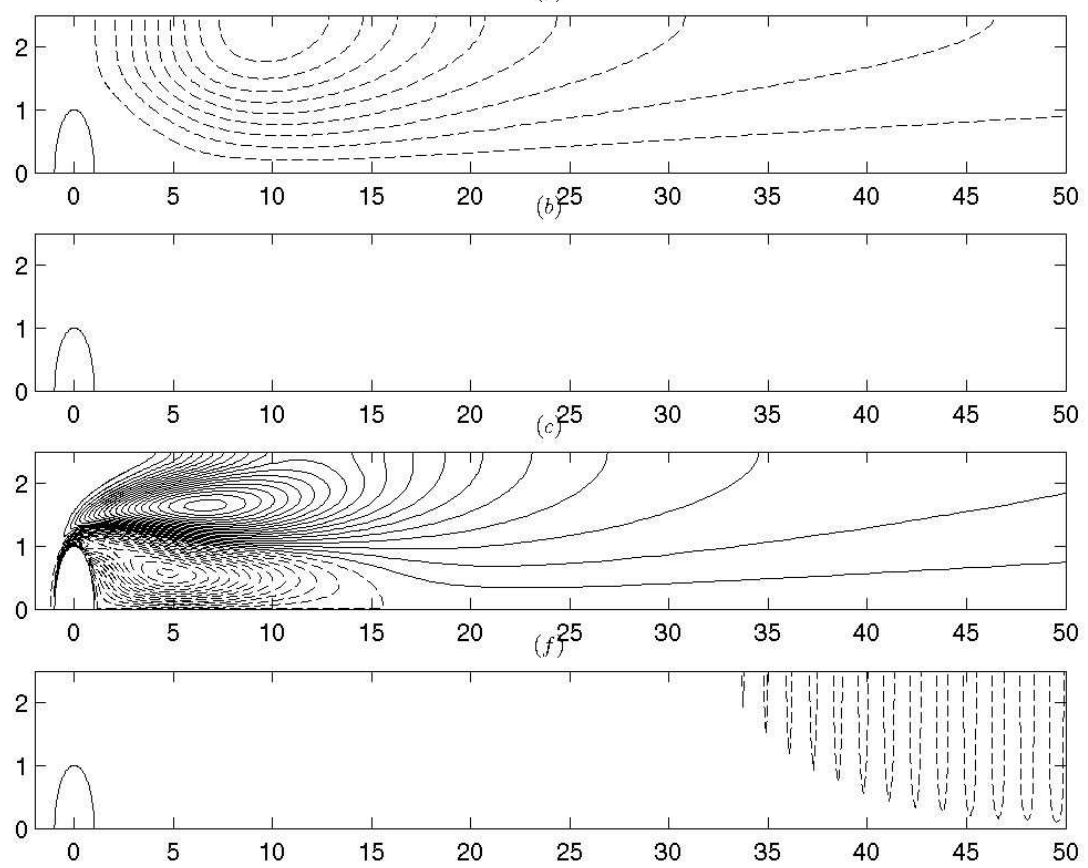

FiguRE 20. Contours of (a) real part of stream function eigenvector, (b) imaginary part of stream function eigenvector, (c) real part of vorticity eigenvector and (d) imaginary part of vorticity eigenvector for $\mathrm{EO}$ modes at $R e_{c}=68.7$ for $W=5, \beta=0.4,(M=641, N=64)$. Note that only the partial domain is shown.

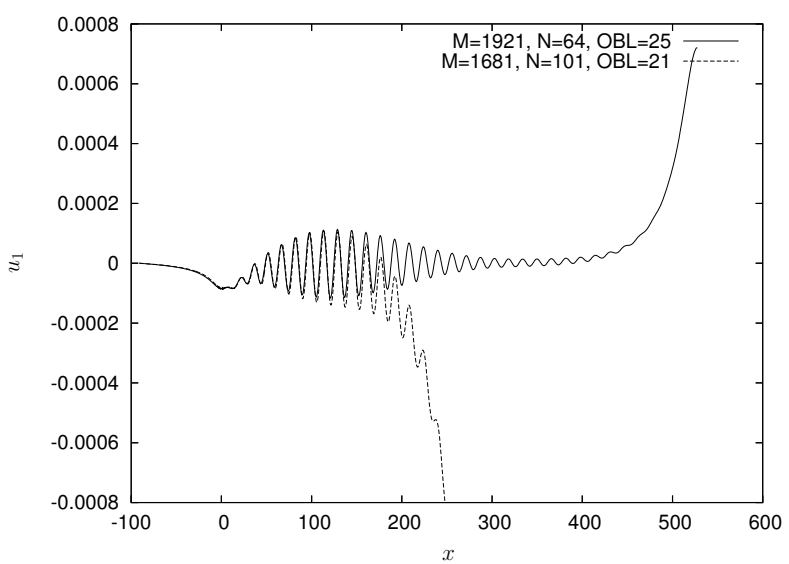

FIGURE 21. Comparing centerline velocity profiles corresponding to $u_{1}$ at $R e_{c} \approx 48.7$ for $W=100 \beta=0.02$, OE modes.

\subsection{Comparison of results with literature}

Table 9 lists the values of critical parameters, $R e_{c}$ and $S t_{c}$ obtained by various researchers. The difference in these values when compared with the literature can be attributed to 


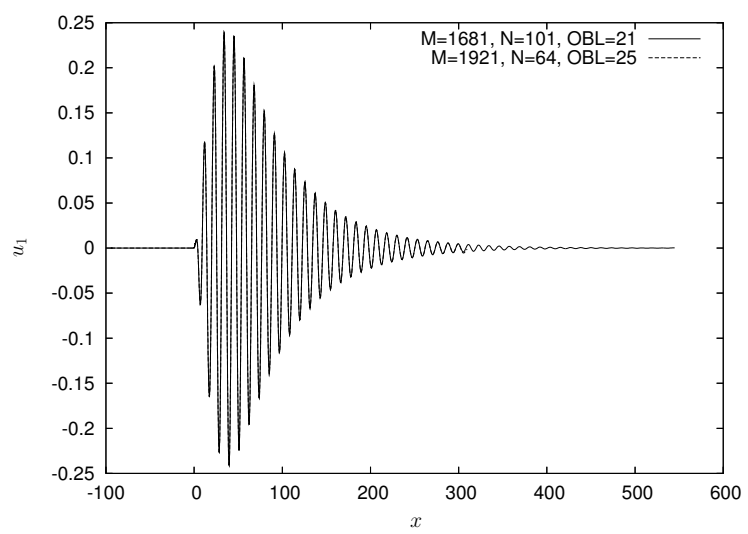

FIGURE 22. Comparing centerline velocity profiles corresponding to $u_{1}$ at $R e_{c} \approx 49.6$ for $W=10 \beta=0.02$, OE modes.

(a)
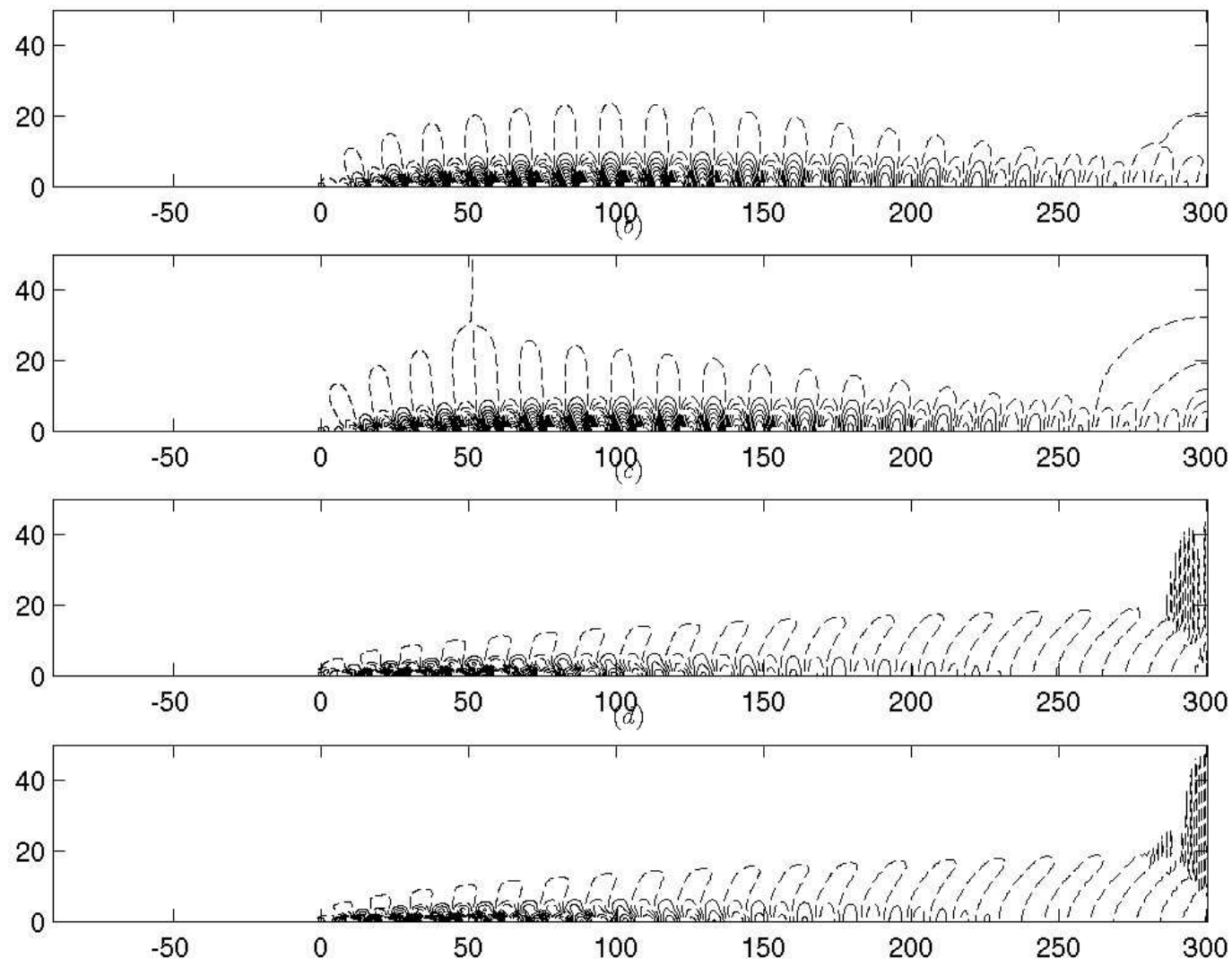

FiguRE 23. Contours of (a) real part of stream function eigenvector, (b) imaginary part of stream function eigenvector, (c) real part of vorticity eigenvector and (d) imaginary part of vorticity eigenvector near the half cylinder for OE modes at $\operatorname{Re}_{c}=48.7$ for $W=100, \beta=0.02$, $(M=1681, N=101$ and $\mathrm{OBL}=21)$. 
(a)
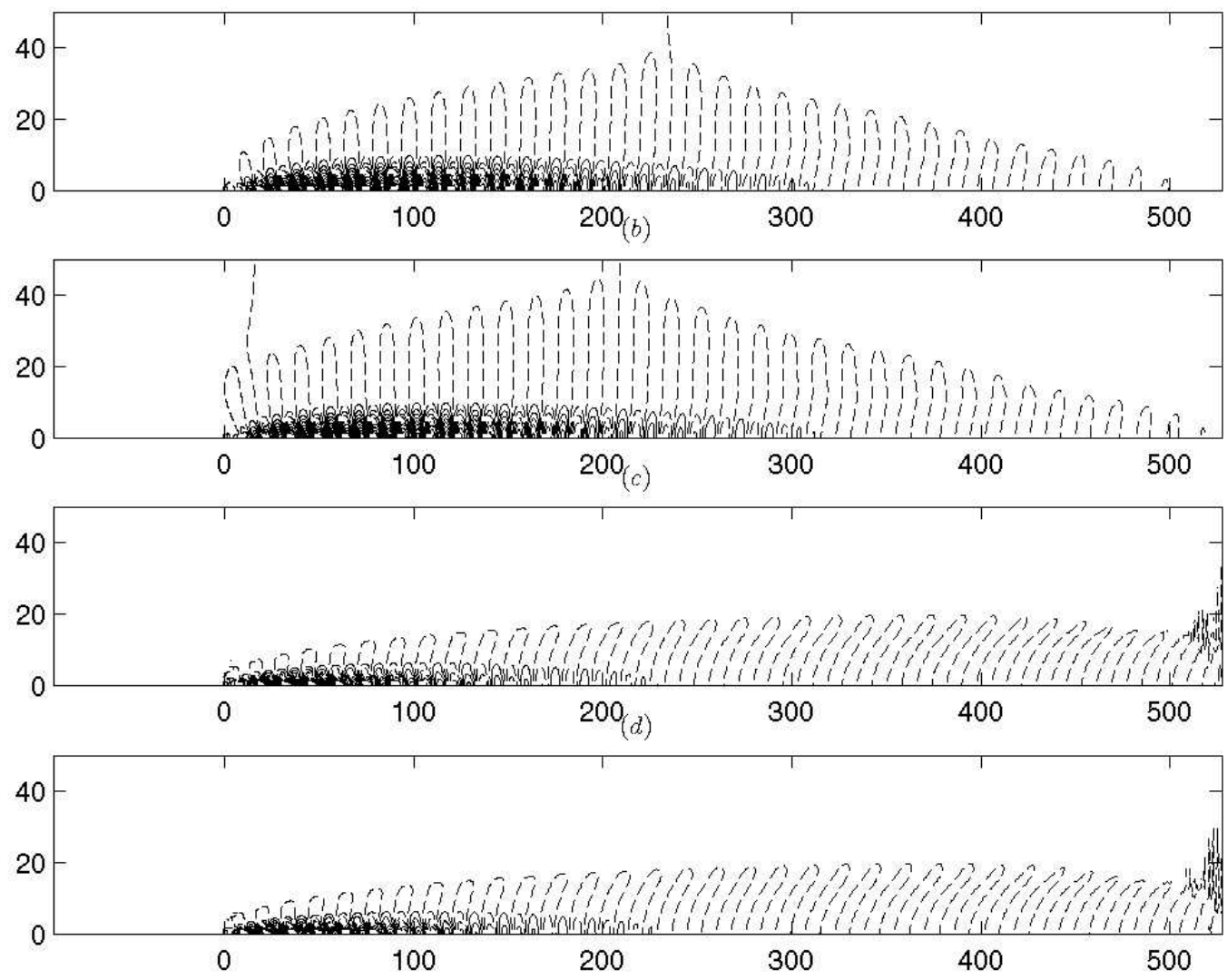

Figure 24. Contours of (a) real part of stream function eigenvector, (b) imaginary part of stream function eigenvector, (c) real part of vorticity eigenvector and (d) imaginary part of vorticity eigenvector for $\mathrm{OE}$ modes at $R e_{c}=48.8$ for $W=100, \beta=0.02,(M=1921, N=64$ and $\mathrm{OBL}=25)$.

different numerical method/experiments adopted for the study, grid size, blockage ratio, location of upstream and downstream boundaries, nature of inlet flow.

The critical values given in the Table 9 for this study correspond to $W=100$ with the blockage ratio $\beta=0.02$ for the $\mathrm{OE}$ modes. This value is chosen because the critical values listed in the table from various studies correspond to unbounded flow past a circular cylinder.

It can be observed from this table that the critical Reynolds number obtained in this study (48.8) is very close to that obtained by Nishioka \& Sato (1978) (48.0) who performed experiments with a blockage of 0.015 . The lowest value of the critical Reynolds number in this table is 34.0 obtained by Coutanceau \& Bouard (1977) for an unbounded case whereas the value reported for the present study correspond to the blockage of 0.02. Similarly, the highest value of the critical Reynolds number in this table $\left(\operatorname{Re}_{c}=54\right)$ is reported by Noack \& Eckelmann (1994) who used global stability analysis of the steady and the periodic cylinder wake by employing a low-dimensional Galerkin method. However, their value is also significantly different from others in the literature. 


\begin{tabular}{ccccccccc}
\hline$\beta$ & \multicolumn{2}{l}{ This study } & \multicolumn{2}{l}{ Kumar \& Mittal (2006) } & \multicolumn{2}{l}{ Sahin \& Owens (2004) } & \multicolumn{2}{c}{ Chen et al. (1995) } \\
\hline & $R e_{c}$ & $S t_{c}$ & $R e_{c}$ & $S t_{c}$ & $R e_{c}$ & $S t_{c}$ & $R e_{c}$ & $S t_{c}$ \\
\hline 0.02 & 48.8 & 0.1181 & 47.19 & 0.1178 & - & - & - & - \\
0.04 & 48.9 & 0.1211 & 46.95 & 0.1206 & - & - & - & - \\
0.1 & 49.8 & 0.1358 & 47.22 & 0.1345 & 50.75 & 0.1211 & 51.6 & 0.122 \\
0.2 & 49.6 & 0.1726 & - & - & 69.35 & 0.1567 & 69.93 & 0.1559 \\
0.4 & 50.8 & 0.2952 & - & - & - & - & 110.1 & 0.286
\end{tabular}

TABLE 10. Comparison of $R e_{c}$ and $S t_{c}$ with literature for various values of blockage ratios, OE modes.

The critical Strouhal number obtained in this study (0.1181) agrees well with Yang \& Zebib (1989) (0.115), Sahin \& Owens (2004) (0.1167) and Kumar \& Mittal (2006) $(0.1167)$ though their corresponding values of $R e_{c}$ are different from our study. While the highest value of $S t_{c}$ in the table is 0.149 obtained by Noack \& Eckelmann (1994), the lowest one is 0.115 obtained by Yang \& Zebib (1989) who used local linear stability theory to investigate the stability characteristics of a circular cylinder wake.

Listed in Table 10 are the critical values compared with the literature for different blockage ratios. Kumar \& Mittal (2006) derived the following relation equation (4.1), for $R e_{c}$ and $S t_{c}$ in terms of blockage based on their numerical experiments in which the upstream and downstream boundaries are set at $50 d$ from the center of cylinder. In our work the downstream boundary for $W=100$ was in excess of $300 d$. It can be seen from centerline velocity plots that at $x=100$ the perturbation has yet to attain its peak values.

$$
\begin{aligned}
R e_{c} & =47.3804-4.70659 \sqrt{\beta}+124.836 \beta-1228.84 \beta^{3 / 2}+4334.98 \beta^{2}-4947 \beta^{5 / 2}, \\
S t_{c} & =0.116311+0.0403825 \beta+1.81145 \beta^{2}-3.97608 \beta^{3} .
\end{aligned}
$$

The values of $R e_{c}$ and $S t_{c}$ in Table 10 by Kumar \& Mittal (2006) are determined using the above equations. It can be observed from this table that the values of the critical Strouhal number are in good agreement (up to 2 decimal digits) with Kumar \& Mittal (2006) though the critical Reynolds number differ for all blockage ratios. The empirical formula shown above may not hold for larger values of $\beta$. The predicted $S t_{c}$ value of 0.1678 for $\beta=0.4$ from (4.1) may be compared with our calculated value of 0.2952 . Also, in their study from $\beta=0.02$ to $0.1, R e_{c}$ values decreased first and then increased while for the same range of $\beta$, our study showed that $R e_{c}$ values are gradually increasing. Interestingly, beyond this value of $\beta$ i.e, for $0.15 \leq \beta \leq 0.4$, our study showed $R e_{c}$ value to be decreasing again and then increasing. Both our study and Kumar \& Mittal (2006)'s study ascertained the fact that the behavior of $R e_{c}$ is non-monotonic with blockage.

For $\beta=0.1,0.2$ and 0.4 , our critical values differ significantly from Sahin \& Owens (2004) and Chen et al. (1995). This is mainly because they have used fully developed flow at the inlet boundary. The experimental findings of Shair et al. (1963) of a $300 \%$ increase in $R e_{c}$ for $\beta=0.2$ as compared to the $\beta=0$ extrapolated value would seem to be at odds with the numerically calculated values of Sahin \& Owens (2004) and Chen et al. (1995). Compared to our results the effect of blockage on the critical Reynolds number by having a uniform flow at the inlet, is marginal, in contrast to that with fully developed flow at the inlet. 


\subsection{Additional comments and comparisons for a pair of cylinders.}

Whilst the single cylinder case has been extensively studied, there are very few results generally available for the flow past a row of cylinders. Williamson (1985) has studied the flow past cylinders experimentally with gap-widths corresponding to $2 \leq W \leq 9$ in our notation. He has found complicated wake vortex shedding patterns which are observed corresponding to in-phase, and anti-phase synchronised, and flip-flop motions. Kang (2003) has been able to reproduce the results in Williamson (1985) but the values of the various parameters used makes it difficult to make any direct comparisons with our results. Most of the computations are for much larger values of the Reynolds numbers than the critical values.

The work of Akinaga \& Mizushima (2005), Mizushima \& Ino (2008) is possibly the closest with respect to the problem studied in this paper. Akinaga \& Mizushima (2005) have used numerical simulations as well as stability analysis to predict the critical Reynolds numbers and Strouhal frequencies. In their work the parameter $\sigma$ is related to our $W$ by $W=4 \sigma+1$ and they find that for the case $\sigma=4, W=17$ the symmetric mode, corresponding to the OE mode studied here, is the most unstable and has critical values $R e_{c}=40.72$ and $S t_{c}=0.128$. For the antisymmetric mode, corresponding to our OO mode, the respective values are $R e_{c}=52.36$ and $S t_{c}=0.127$. If we compare the results for $W=20$ given in Tables $3,5 R e_{c}$ is 49.8 and 46.8 for the OE and OO modes respectively, whereas the critical Strouhal values are $S t_{c}=0.1358$ and $S t_{c}=0.06731$, which are very different as compared to Akinaga \& Mizushima (2005). In figure 4 it can be seen that the $\mathrm{OO}$ mode is the more unstable for blockage ratios in the vicinity of $\beta=0.1$, corresponding to $W=20$, which is opposite to that found by Akinaga \& Mizushima (2005). Also Akinaga \& Mizushima (2005) find that there is a crossover of instabilities at a value of $\sigma=2.34$ corresponding to $W=10.34$ and a blockage ratio of $\beta=0.193$. Figure 4 shows that there is a crossover of instabilities at a similar value of $\beta \sim 0.19$ but whereas in Akinaga \& Mizushima (2005) for $\beta>0.1932$ the OO mode is more unstable, our conclusions are opposite in that in this range we find the $O E$ mode to be the more unstable. One possible reason for such a large discrepancy in the results may be due to the values of the outer boundary locations which is set at 30 cylinder diameters in Akinaga \& Mizushima (2005), as compared to several hundred in our work. Peschard \& Le Gal (1996) suggested that the the anti-phase and in-phase oscillatory motion could be described by a coupled oscillator model, but this was dismissed by Akinaga \& Mizushima (2005) on the grounds that the critical frequencies for the two modes were found to be very similar. However in our results the two critical frequencies are quite distinct. In their experiments Peschard \& Le Gal (1996) find for large gap widths $W>6$ that there is no coupling between the two vortex streets, but for smaller gap-widths there are much more complicated interactions. Again, the Reynolds number regime studied here is far above the critical values.

\section{Conclusions}

The instability of flow past a row of circular cylinders has been examined for various blockage ratios, extending previous work by Fornberg (1991) and Gajjar \& Azzam (2004) for the steady case. The same numerical techniques as used in Gajjar \& Azzam (2004) to compute the steady flow for large Reynolds numbers, have been adapted for the unsteady case and the critical parameters for the onset of instability have been determined. It is found that there are 3 unstable mode classes and the primary wake instability is due to a Hopf bifurcation to the $\mathrm{OE}$ and $\mathrm{OO}$ modes. The OE modes correspond to antiphase 
oscillatory motion in which the disturbance streamfunction is anti-symmetric about the mid-plane between the cylinders. The OO modes correspond to in-phase oscillatory motion in which the disturbance streamfunction is symmetric about the same mid-plane. In both cases the disturbance streamfunction is symmetric with respect to the wake centerline passing through the cylinder centres. For the flow past a single cylinder results for the OE modes are in good agreement with those published extensively in the literature. It is found that for low blockage ratios, the OO modes correspond to low frequency oscillations and are the first to become unstable although the critical Reynolds numbers for instability are not too different for the $\mathrm{OO}$ and $\mathrm{OE}$ modes.

The critical parameters have been found to be largely insensitive to the grid sizes and parameters used in this study for values of the gap width $W=5,10,20,50$ corresponding to blockage ratios of $\beta=0.4,0.2,0.1$ and $\beta=0.04$. For the larger gap width case of $W=100(\beta=0.02)$, the values of $R e_{c}$ are found to be fluctuating between 48.5 and 48.8 depending on the value of location of the outlet boundary, whereas the values of the critical Strouhal number $S t_{c}$ is found to be insensitive. This fluctuation of values may be taken care of by either increasing the value of the outlet boundary location or by using different (non-reflective) downstream boundary conditions.

The numerical results obtained have shown that the positioning of the downstream boundary can affect the critical values obtained and that for small blockage ratios the downstream boundary needs to be much further than for large blockage ratios. This means that the study of the instability of flow past an isolated circular cylinder requires not only good resolution near the cylinder but also very long domains. This is one of the reasons why such computations are difficult to perform accurately.

An attempt was also made to study the influence of blockage on the critical values. It was found that the critical Strouhal number exhibits a monotonic behavior with blockage while the critical Reynolds number do not. In addition centerline perturbation velocities for the OE modes are significantly increased with increasing blockage. Kumar et. al. (2009) have suggested that this non-monotonic variation is because of the effect of the lateral boundaries in suppressing disturbance growth in the cross-stream direction, together with the effect of local flow acceleration in the streamwise direction.

For the flow past a single cylinder, the effect of blockage on the critical parameters with a fully developed inlet profile is much more pronounced than with a uniform flow at the inlet. In both cases blockage increases the critical Reynolds number for instability, although our results indicate that the the increase is only marginal for the latter.

Our study has also shown that beyond the primary instability there are other unstable modes and in particular for $W=20, \beta=0.1$ and $W=50, \beta=0.04$ a second unstable pair of eigenvalues can be found for the OE mode at Reynolds numbers between 125 and 150. This raises doubts about the comment made in Barkley \& Henderson (1996) that 'there are no further two-dimensional bifurcations in the flow dynamics' before the three-dimensional mode at a higher Reynolds number.

The few published papers on the instability of flow past a row of cylinders have shown that there exists a rich dynamics of different vortex induced motions, both experimentally and numerically, but there appear to be very few detailed numerical results, certainly in the parameter regimes studied in the current paper. With the one study where it was possible to make some comparisons, we find strong disagreement as far as the critical Strouhal frequencies and Reynolds numbers are concerned. It is noted that our results for the OO modes point to a low frequency oscillation, and such types of oscillations arise in other contexts involving separated bubbles and wake instabilities, see Castro (2005) for example and the references therein. The low frequency oscillations have not been properly understood and it would be intriguing if these are connected with the 
OO modes that we have discussed. The eigenfunctions for both modes have very similar features and as the coupled oscillator model of Peschard \& Le Gal (1996) shows there are distinct possibilities of intricate interchanges between the different states.

There have been many numerical investigations of hydrodynamic instabilities based on solving the generalised eigenvalue problem using subspace iteration. The study of instabilities in a lid-driven cavity in Boppana \& Gajjar (2010) and others, suggests that conclusions based on just a global stability analysis can sometimes be inadequate, and should ideally be cross-checked with simulations of the linearised unsteady equations, something admittedly not done here. In Boppana \& Gajjar (2010) it was found that working with a small basis and certain shift parameters, it was indeed possible to miss physically more unstable modes which were present. It is noted that the number of basis vectors used in many studies is small as otherwise computational times can be prohibitively excessive. In the current work it has been possible to do proper grid size studies because of the numerical method which has been used.

It would be interesting also to explore the further nonlinear development of the instability and three-dimensional aspects. The former necessitates working with the full physical domain and not assuming symmetry as we have done. This of course requires a considerable non-trivial modification to the techniques used in this study.

\subsection{Acknowledgements}

The referees are thanked for their helpful comments, and in particular the authors are extremely grateful to one anonymous referee who suggested extending the calculations to the OO, and EO modes and for pointing out the link between the various modes and the in-phase and anti-phase oscillatory motions. VBLB is grateful to the School of Mathematics of Manchester University for financial support, and JSGB is grateful to the EPSRC for partial support under a grant EP/D050847/1.

\section{REFERENCES}

Adbessemed, N., Sharma, A., Sherwin, S. And Theofilis, V. 2009 Phys. Fluids, 21, 044103.

Akinaga, T. And Mizushima, J. 2005 Linear stability of flow past two cylinders in a sideby-side arrangement. J. Phy. Soc. Japan, 74, 1366-1369.

Barkley, D. And Henderson, R. D. (1996) Three-dimensional Floquet stability analysis of the wake of a circular cylinder. J. Fluid Mech., 322, 215-241.

Barkley, D., Blackburn, H. M. And Sherwin, S. J. 2008 Direct growth analysis for timesteppers. Int. J, Numer. Fluids, 57, 1435-1458.

Behr, M., Liou, J., Shih, R. And Tezduyar, T. E. 1991 Vorticity-Streamfunction formulation of unsteady incompressible flow past a cylinder: Sensitivity of the computed flow field to the location of the outflow boundary. Int. J. Numer. Fluids, 12, 323-342.

Blackburn, H. M., Sherwin, S. J. And Barkley, D. 2008 Convective instability and transient growth in steady and pulsatile stenotic flows. J. Fluid Mech., 607, 267-277.

Boppana, V. B. L. 2007 Flow instability in a lid-driven cavity and circular cylinder cascade. Univ. of Manchester, Ph. D. thesis.

Boppana, V. B. L. And GajJar, J. S. B. 2010 Global flow instability in a lid-driven cavity. Int. J. Numer. Fluids, 62, 827-853.

Cadou, J. M., Potier-Ferry, M. And Cochelin, B. 2006 A numerical method for the computation of bifurcation points in fluid mechanics. Eur. J. Mech. B Fluids, 25, 234-254.

Camarri, S. And Giannetti, F. 2007 On the inversion of the von Kármán street in the wake of a confined square cylinder. J. Fluid Mech., 574, 169-178.

Canuto, C. A., Hussaini, M. Y. And Zang, T. 1987 Spectral methods in Fluid Dynamics.Springer Series in Computational Physics, Springer-Verlag.

Castro, I. P. 2005 The stability of laminar symmetric wakes. J. Fluid Mech., 532, 389-411. 
Chen, J. H., Pritchard, W. G. and Tavener, S. J. 1995 Bifurcation for flow past a cylinder between parallel planes. J. Fluid Mech., 284, 23-41.

Coutanceau, M., And Bouard, R. 1977 Experimental determination of the main features of the viscous flow in the wake of a circular cylinder in uniform translation. Part 1. Steady flow. J. Fluid Mech., 79, 231-256.

Coutanceau, M. and Defaye, J. R. 1991 Circular cylinder wake configurations. Appl. Mech. Rev., 44, 255-305.

Davies, C. And CARPenter, P. W. 2003 Global behaviour corresponding to absolute instability of the rotating-disc boundary layer. J. Fluid Mech., 486, 287-329.

Ding, Y. 2003 Computation of leading eigenvalues and eigenvectors in the linearized NavierStokes equations using Krylov subspace method. Int. Jour. of Comp. Fluid Dyn., 17 (4), 327-337.

Ding, Y. And KaWAhara, M. 1999 Three-dimensional linear stability analysis of incompressible viscous flows using the finite element method. Int. J. Numer. Meth. Fluids, 31, $451-479$.

FASEL, H. F. 1976 Investigation of the stability of boundary layers by a finite-difference model of the Navier-Stokes equations. J. Fluid Mech., 78, 355-383.

Fornberg, B. 1985 Steady viscous flow past a circular cylinder up to Reynolds number 600. J. Comp. Phy., 61, 297-320.

Fornberg, B. 1991 Steady incompressible flow past a row of circular cylinders. J. Fluid Mech., 225, 655-671.

GajJar, J. S. B. And Azzam, N. A. 2004 Numerical solution of the Navier-Stokes equations for the flow in a cylinder cascade. J. Fluid Mech., 520, 51-82.

Gallaire, F., Marquille, M. and Ehrenstein, U. 2007 Three-dimensional tranverse instabilities in detached boundary layers. J. Fluid Mech., 571, 221-233.

Goujan-Durand, S., JenfFer, P. And Wesfreid, J. E. 1994 Downstream evolution of the Bénard-von Kármán instability. Phy, Rev. E., 50(1), 308-313.

Gresho, P. M. , Chan, S. T. , Lee, R. L. And Upson, C. D. 1984 A modified finite element method for solving the time-dependent, incompressible Navier-Stokes equations. Part2: Applications. Int. J. Numer. Fluids, 4, 619-640.

Henderson, R. D. 1995 Details of the drag curve near the onset of vortex shedding. Phys. Fluids, 7 (9), 2102-2104.

Hultgren, L. And Aggarwal, A. K. 1987 Absolute instability of the Gaussian wake profiles. Phy. Fluids, 30, 3383-3387.

JACKson, C. P. 1987 A finite-element study of the onset of vortex shedding in flow past variously shaped bodies. J. Fluid Mech., 182, 23-45.

KANG, S. 2003 Characteristics of flow over two circular cylinders in a side-by-side arrangement at low Reynolds numbers. Phys. Fluids, 15(9), 2486-2498.

KovÁsznay, L. S. G. 1949 Hot-wire investigation of the wake behind cylinders at low Reynolds numbers. Proc. Roy. Soc. of London. Series A. Mathematical and Physical Sciences, 198 (1053), 174-190.

Kumar, B. And MitTal, S. 2006 Effect of blockage on critical parameters for flow past a circular cylinder. Int. J. Numer. Fluids, 50, 987-1001.

Kumar, B., Kottaram, J. J., Singh, A. K. And Mittal, S. 2009 J. Fluid. Mech., 632, 273-300.

LehoucQ, R. B., Sorensen, D. C. And YAng, C. 1988 ARPACK User's guide, Solution of large-scale eigenvalue problems with implicitly restarted Arnoldi methods. SIAM.

Lingwood, R. J. 1995 Absolute instability of the boundary layer on a rotating-disk. J. Fluid Mech., 299, 17-33.

Marquet, O., Sipp, D., Chomaz, J. M. And Jacquin, L. 2008 Amplifier and resonator dynamics of a low-Reynolds-number recirculation bubble in a global framework. J. Fluid Mech., 605, 429-443.

Marquille, M. And Ehrenstein, U. 2003 On the onset of nonlinear oscillations in a separating boundary-layer flow. J. Fluid Mech., 490, 169-188.

Mittal, S., Kottaram, J. J. And Kumar, B. 2008 Onset of shear layer instability in flow past a cylinder. Phys. Fluids, 20, 054102-1-10. 
Mizushima, J., AND Ino, Y. 2008 Stability of flows past a pair of circular cylinders in a side-by-side arrangement. J. Fluid Mech., 595, 491-507.

Morzyński, M. AND Thiele, F. 1991 Numerical stability analysis of a flow about a cylinder. Z. angew. Math. Mech., 71 (5), 424-428.

Morzyński, M., Afanasiev, K. And Thiele, F. 1999 Solution of the eigenvalue problems resulting from global non-parallel flow stability analysis. Comput. Methods Appl. Mech. Eng., 169, 161-176.

NishiokA, M. AND SATo, H. 1978 Mechanism of determination of the shedding frequency of vortices behind a cylinder at low Reynolds numbers. J. Fluid Mech., 89, 49-60.

NoAck, B. R. And Eckelmann, H. 1994 A global stability analysis of the steady and periodic cylinder wake. J. Fluid Mech., 270, 297-330.

Peschard, I. And Le Gal, P. 1996 Coupled Wakes of Cylinders. Phy. Rev. Lett., 77, 31223125.

PIER, B. 2002 On the frequency selection of finite-amplitude vortex shedding in the cylinder wake. J. Fluid Mech., 458, 407-417.

Pier, B. AND Huerre, P. 2001 Nonlinear self-sustained structures and fronts in spatially developing wake flows. J. Fluid Mech., 435, 145-174.

PIER, B. 2008 Local and global instabilities in the wake of a sphere. J. Fluid Mech., 603, 39-61.

SAAD, Y. 1989. Numerical solution of large nonsymmetric eigenvalue problems. Comp. Phy. Comm., 53, 71-90.

Sahin, M. And Owens, R. G. 2004 A Numerical Investigation of Wall Effects up to High Blockage Ratios on Two-Dimensional Flow Past a Confined Circular Cylinder. Physics of Fluids, 16 (5), 1305-1320.

Shair, F. H., Grove, A. S., Petersen, E. E. And Acrivos, A. 1963 The effect of confining walls on the stability of the steady wake behind a circular cylinder. J. Fluid Mech., 17, $546-550$.

Strykowski, P. J. and Hannemann, K. 1991 Temporal simulation of the wake behind a circular cylinder in the neighborhood of the critical Reynolds number. Acta Mechanica, 90, $1-20$.

TheofiLis, V. 2003 Advances in global linear instability analysis of nonparallel and threedimensional flows. Prog. in Aero. Sci., 39, 249-315.

Tuckerman, L. S. AND BARkLey, D. 2000 Bifurcation analysis for timesteppers. Numerical Methods for Bifurcation Problems and Large-Scale Dynamical Systems. Doedel, E. and Tuckerman, L. S. (eds). Springer: Berlin, 453-566.

Williamson, C. H. K. 1985 Sinusoidal flow relative to circular cylinders. J. Fluid Mech., 155, 141-174.

Williamson, C. H. K. 1989 Oblique and parallel modes of vortex shedding in the wake of a circular cylinder at low Reynolds numbers. J. Fluid Mech., 206, 579-627.

Williamson, C. H. K. 1996 Vortex dynamics in the cylinder wake. Ann. Rev. Fluid Mech., 28, 477-539.

YANG, X. AND ZEBIB, A. 1989 Absolute and convective instability of a cylinder wake. Phys. Fluids A, 1 (4), 689-696.

Zebib, A. 1987 Stability of viscous flow past a circular cylinder. Jour. of Eng. Math., 21, 155-165. 